\title{
Experimental identification of social learning in wild animals
}

\author{
Simon M. READER \\ Utrecht University, Utrecht, The Netherlands \\ AND \\ DORA BIRO \\ University of Oxford, Oxford, England
}

\begin{abstract}
Field experiments can provide compelling demonstrations of social learning in wild populations. Social learning has been experimentally demonstrated in at least 23 field experiments, in 20 species, covering a range of contexts, such as foraging preferences and techniques, habitat choice, and predator avoidance. We review experimental approaches taken in the field and with wild animals brought into captivity and note how these approaches can be extended. Relocating individuals, introducing trained individual demonstrators or novel behaviors into a population, or providing demonstrator-manipulated artifacts can establish whether and how a particular act can be socially transmitted in the wild and can help elucidate the benefits of social learning. The type, strength, and consistency of presented social information can be varied, and the provision of conditions favoring the performance of an act can both establish individual discovery rates and help determine whether social information is needed for acquisition. By blocking particular avenues of social transmission or removing key individuals, routes of transmission in wild populations can be investigated. Manipulation of conditions proposed to favor social learning can test mathematical models of the evolution of social learning. We illustrate how field experiments are a viable, vital, and informative approach to the study of social learning.
\end{abstract}

Social learning is taxonomically widespread, with both invertebrates and vertebrates acquiring information from either the behavior of other individuals or the products of their behavior (Galef \& Laland, 2005; Leadbeater \& Chittka, 2007). Social learning has been demonstrated in multiple behavioral contexts of adaptive significance, such as the learning of foraging resources, foraging techniques, settlement locations, travel routes, predator responses, and mate preferences (Galef \& Laland, 2005; Griffin, 2004; Leadbeater \& Chittka, 2007). Moreover, social learning underlies the emergence and maintenance of cultures (Boyd \& Richerson, 1985). Thus, the mechanisms underlying social learning, their evolution and development, and their adaptive consequences are all important topics relevant to a range of fields, including ethology, ecology, psychology, anthropology, and evolutionary biology. Here, we argue that field studies are a vital but underused part of answering questions about these topics. Field experiments complement and extend other approaches to the study of social learning and should not be isolated from them.

Multiple processes underlie social learning, and research into social learning is not characterized by a single question (Galef, 1988; Heyes, 1994). Consequently, any single approach will be inadequate to provide a complete explanation of the causes and consequences of socially learned behaviors. Observational and experimental studies in the laboratory and the wild, combined with theoretical and even archeological approaches, are all important and valuable methodologies (Dawkins, 2007; Franz \& Nunn, 2009; Haslam et al., 2009; Kendal, Kendal, Hoppitt, \& Laland, 2009; Lefebvre, 1995; Reader, 2004; van Schaik et al., 2003; Whiten et al., 1999; see also the contents of this special issue: Kendal, Galef, \& van Schaik, 2010). Particularly valuable are cycles of feedback between experiments in captivity and those in the field, as is experimental study in the laboratory of behavior patterns observed in the wild and ascribed to social learning. We describe such examples below, alongside pure field experiments.

The value of field experiments. Use of manipulative experimental approaches with wild populations is relatively new in the field of social learning, possibly fueled by the recent explosion of interest in nonhuman animal cultures (Fragaszy \& Perry, 2003; Heyes \& Galef, 1996; Laland \& Galef, 2009). As was pointed out most emphatically by Laland and colleagues (Laland \& Hoppitt, 2003; Laland \& Janik, 2006) but has been a point made repeatedly over the history of social-learning research (e.g., Galef, 1976), a lack of direct evidence for social learn- 
ing's maintaining community-specific traditions in the wild represents a significant stumbling block for research, particularly for assessment of the importance of social learning in natural circumstances. Population differences in behavioral repertoires have been ascribed to differential innovation being followed by social learning within a locality, with the method of exclusion used to rule out genetic or ecological explanations for population differences (e.g., van Schaik et al., 2003; Whiten et al., 1999). However, ruling out both ecological and genetic factors as alternative explanations is considered both highly problematic and logically insufficient by many commentators (Laland \& Galef, 2009). Moreover, since social learning allows animals to acquire behavior appropriate to local conditions, perhaps the most important instances of social learning will be those linked to local ecology - the very examples ruled out by the method of exclusion. Controlled experimental manipulations, allowing convincing demonstrations of social influences on learning, are thus fundamental to confirming the existence of animal cultures and social learning, even when such experiments do not elucidate the precise social-learning mechanisms underlying animal traditions. Given the vast corpus of work examining social-learning processes in the laboratory that has provided a suite of useful approaches, field studies have much catching up to do. Yet their value is potentially immense.

Field experiments can demonstrate that social learning operates in free-living animal populations. Without such data, the most elegant laboratory demonstrations of social learning will fail to confirm that the same mechanisms are employed in the normal life of animals, thus leaving opaque the relevance and relation of the results of laboratory studies to ecology and to the evolution of social information use (Galef, 1976; Seppänen \& Forsman, 2007). Unidentified features of field environments or field-rearing conditions may alter experimental outcomes (Cuthill, 1991). Social learning may be employed only in the idealized conditions of the laboratory, or, conversely, animals may be able to acquire behavior patterns individually in the laboratory but, in the wild, employ social learning for the same purpose. For example, alternative acquisition routes available in the wild may facilitate social learning, such as multiple demonstrators or demonstrators in long-lasting close proximity (e.g., mothers and infants).

Animals in the field have varied options available to them, whereas in the laboratory, the social and physical environment is typically more impoverished and options are more restricted. For example, foraging free-living animals could maintain established, rewarded behavior even when social information indicates that alternatives are available, whereas social learning might be the only route to food in a captive setting. Field studies also allow the diffusion (or lack thereof) of a behavior pattern to be monitored, and such diffusion may differ from expectations based on either payoffs resulting from engaging in a behavior pattern or patterns of diffusion observed in the laboratory (Kummer \& Goodall, 1985; Laland \& Hoppitt, 2003; Lefebvre \& Palameta, 1988; Reader \&
Laland, 2003b). Possible causes of such differences in the patterns of diffusion observed in the laboratory and in nature could derive from the structure of social networks and patterns of interactions or from nondemonstrators influencing social learning. For example, bystanders may distract observers or disrupt demonstrators (Lefebvre \& Giraldeau, 1994).

On a heuristic level, the constraints of field research can be useful in simplifying research questions and experimental designs to address core issues, and fieldwork can sharpen the relevance and methodology of laboratory experiments. Field experiments thus provide the opportunity to establish the mechanisms and benefits of social learning in wild populations, allowing researchers to go beyond the question "Is social information utilized?" and to examine a number of issues of applied and theoretical interest. Given the limitations of the field in the majority of study systems (including varied ethical and practical considerations; Cuthill, 1991), experiments that replicate the exacting control possible in the laboratory are difficult to devise. However, rigorous demonstrations of social learning, underlying processes, and adaptive consequences are feasible in the field. Below, we summarize approaches that have moved beyond observational data by employing some ingenious methods to demonstrate the occurrence of socially mediated learning among wild animals.

What are field experiments? We suspect that all readers will have an intuitive understanding of both field and experiment, but for clarity, we provide the operational definition that we used in our survey of the published social-learning literature: manipulations of free-living populations. These manipulations are typically transient or limited modifications of either well-defined habitat or individual characteristics. We do not include studies involving gross manipulations, such as cross-fostering members of one species to another, because in such cases the causal role of social information will be difficult to determine (Slagsvold \& Wiebe, 2007). We exclude from our survey natural experiments, since manipulations are uncontrolled and are not performed by an experimenter, although we mention particular illustrative cases below.

Our delimitation between field and captive studies has arbitrary characteristics. For example, free-living animals may reside in areas impacted or provisioned by humans, resulting in effects on their behavior and evolution (McDougall, Réale, Sol, \& Reader, 2006). Indeed, social learning may be a key part of allowing animals to cope with human impacts (Lee, 1991; Reader \& Laland, 2003a; Whitehead, 2010). Moreover, field experiments may involve arbitrary or artificial stimuli or domesticated animal strains, whereas captive studies may use wild-caught animals or may closely recreate native environments. We strongly encourage captive studies using naturalistic environments, which provide many advantages over artificial captive environments. We exclude field experiments in which animals are not free-living at the time of testing, such as wild individuals placed in temporary enclosures in the field (e.g., the escape-route learning tests of Reader, 
Kendal, \& Laland, 2003), even though such studies use individuals with natural experiences up to the point of testing. We suggest that our focus on free-living populations avoids an overly restrictive survey and captures the key feature that manipulations are conducted within a rich physical and social environment, with multiple options available to the animals.

Summary of aims. We have four aims: (1) to survey existing work; (2) to illustrate the advantages and disadvantages of current approaches; (3) to detail the essential requirements to confirm social learning; and (4) by including discussion of captive studies particularly relevant to fieldwork, to suggest additional or alternative approaches. On any criteria, demonstrations of social learning in wild populations are rare. We hope to encourage field research into a variety of questions concerning social learning.

\section{Field Experiments}

The survey. Our survey (Table 1) covers social learning in nonhuman animals, excluding examples of social information use where learning has not been definitively demonstrated. Thus, we exclude examples in which behavior has not been measured in the absence of either the producers of social information (henceforth, demonstrators) or their artifacts. For example, mate-choice copying (a form of social information use) was demonstrated in wild sailfin mollies (Poecilia latipinna), but the design did not incorporate a subsequent phase without demonstrators to test whether any learning had taken place (Witte \& Ryan, 2002). Similarly, Hromada, Antczak, Valone, and Tryjanowski (2008) demonstrated that male red-backed shrikes (Lanius collurio) used the social information provided by food caches (impaled prey, artifacts of successful foraging) in making settlement decisions, but the authors did not observe behavior in the absence of the caches. We suspect that learning is likely in such cases, but it was not formally demonstrated.

We exclude cases of social information use without learning, not because we believe that it is unimportant or uninteresting, but because we believe that social learning is qualitatively different from social information use without learning. Although there are cases in which social information will be either long-lasting (e.g., animal products) or consistently available, learned behavior can be maintained after a social cue has been removed and behavior has been interrupted (Boyd \& Richerson, 1985; Morand-Ferron, Doligez, Dall, \& Reader, in press). For example, knowledgeable conspecifics mobbing a predator may be joined by a naive individual that continues mobbing when the conspecifics stop. However, if no learning has occurred, the naive individual will not respond to the predator if it reappears (Curio, Ulrich, \& Vieth, 1978).

We roughly classify examples according to the nature of the principal manipulations performed: (1) manipulating physical aspects of the environment, (2) manipulating individual behavior (including manipulation of individual presence), (3) manipulating transmission routes, or (4) some combination of these. We also focus on the need for adequate control conditions to eliminate alter- nate explanations of social learning. We include in our survey social-learning field experiments producing negative results. The value of such experiments lies in demonstrating a lack of a need for social learning, particularly when purely observational data are suggestive of, and are readily interpreted as, illustrating behavioral traditions (Laland \& Janik, 2006).

A recent review (Galef, 2004) highlighted field experiments as a promising approach to the study of traditional behaviors but listed only five such examples, three of which we would exclude from our survey (one was arguably nonmanipulative according to our definition, ${ }^{1}$ another did not specifically test social learning, ${ }^{2}$ and the third did not incorporate a control condition allowing comparisons of individual and social components of learning ${ }^{3}$ ). Our survey, unlikely to be exhaustive, expands considerably on this review but reveals that field experiments are still relatively rare. We document 23 studies, most published since Galef's (2004) review, covering 20 species, that demonstrate social learning in the field and 3 studies that provide no evidence for social learning. These important findings confirm that field experiments are viable and can inform us regarding social learning in natural contexts.

Manipulating the environment or populations wholesale: Coral reef fish translocation studies. In the most dramatic manipulations of the environment, the ground is figuratively pulled from under a population's feet, since its members are moved from their original range into a novel setting. If behavior patterns are maintained by social learning, rather than being channeled by some ecological constraint, moving an entire population into a new environment allows three predictions. First, if social learning is involved, the translocated population should, so far as possible, maintain its established traditions. Second, if the new location was previously home to another, now absent, population, the new arrivals will not necessarily adopt the traditions of the individuals that previously lived there. Third, if the new locale contains suitable demonstrators (e.g., members of the local population who exhibit a putative tradition), translocated individuals may acquire any putatively traditional behaviors that these local "demonstrators" exhibit. All three of these predictions hold true in the case of spatial behavior in coral reef fish.

Helfman and Schultz (1984) demonstrated that the third of these principles operates in free-living French grunts (Haemulon flavolineatum). Individual fish experimentally displaced to novel locations followed the native population on their daily travel routes toward shoaling sites and subsequently adopted these routes themselves when traveling alone, thus demonstrating social learning, in addition to social information use when demonstrators were present. Importantly, control fish, translocated to the same location after the native population had been removed and, thus, lacking opportunities to follow demonstrators, did not adopt the routes previously used by the removed individuals. These experimental data imply that daily shoaling sites were maintained via socially transmitted information between knowledgeable demonstrators 


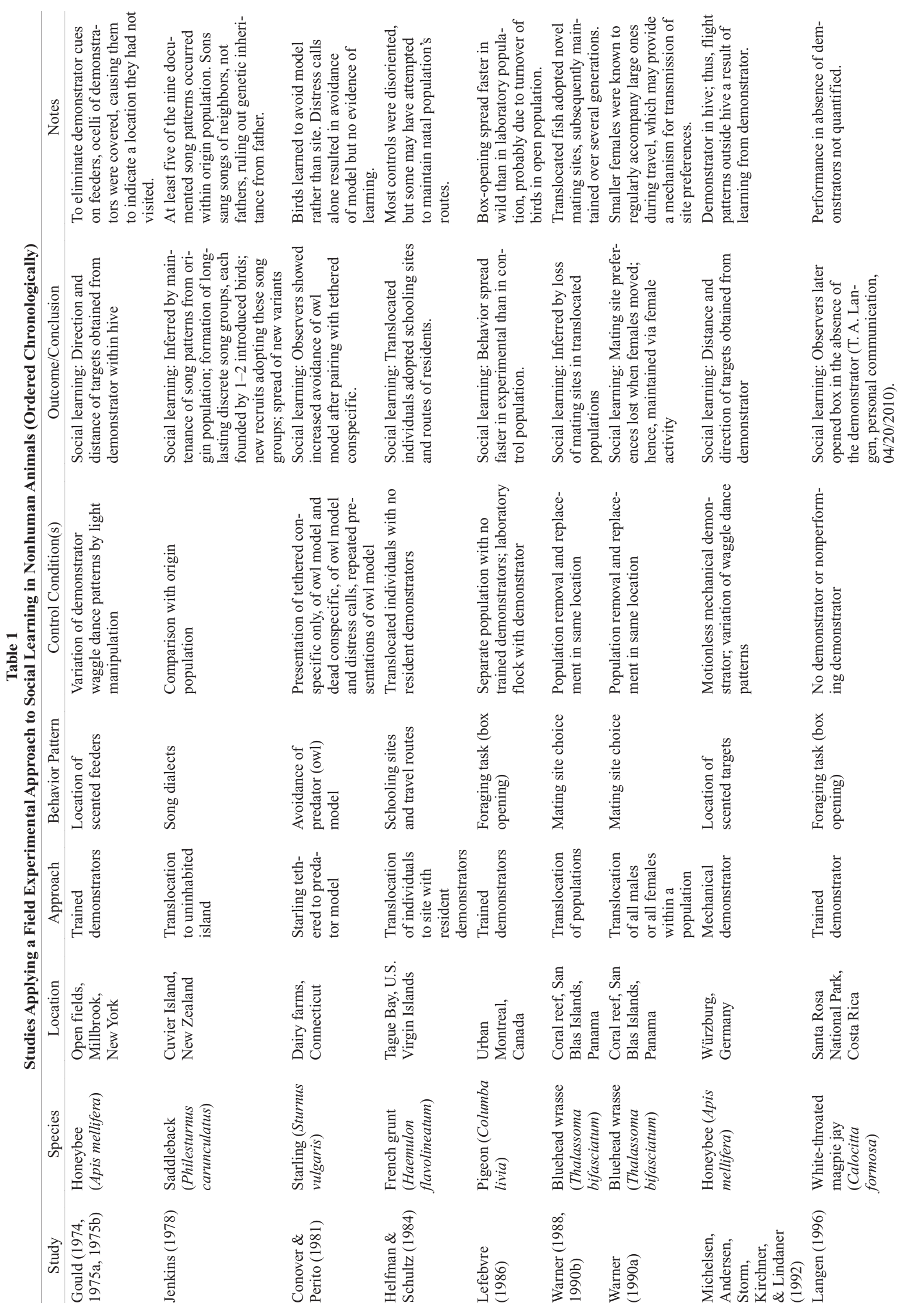



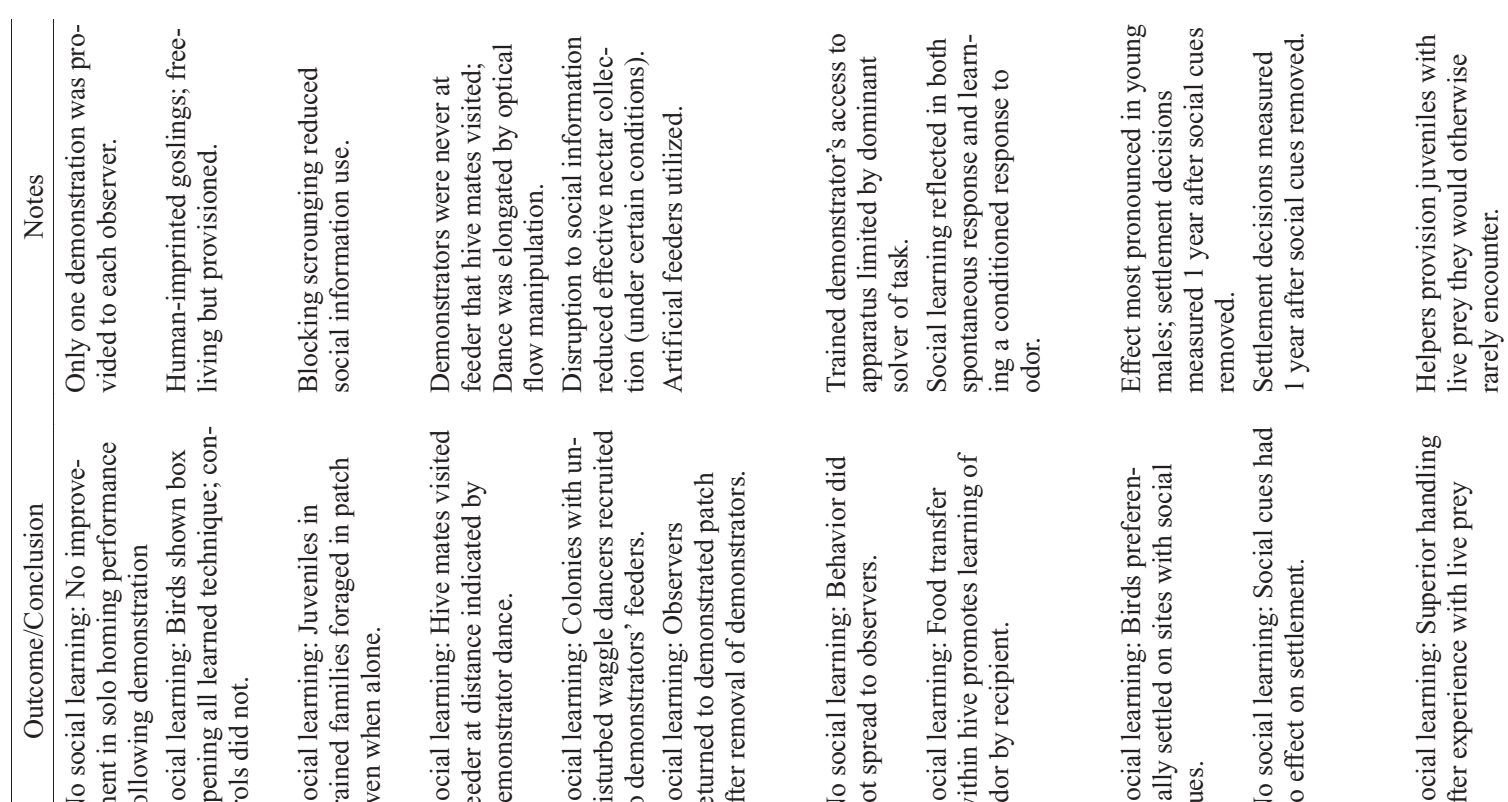

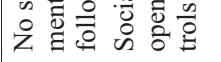

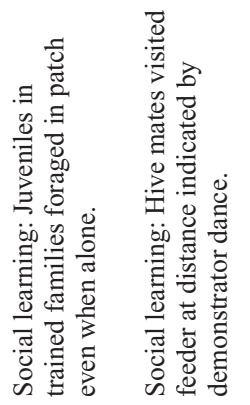

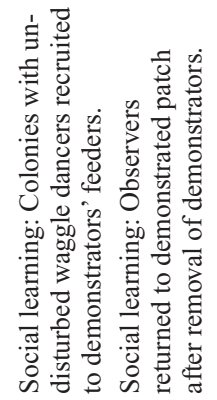

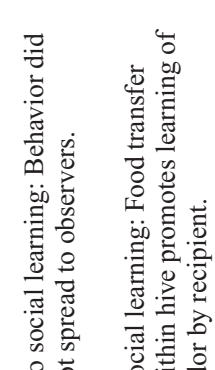

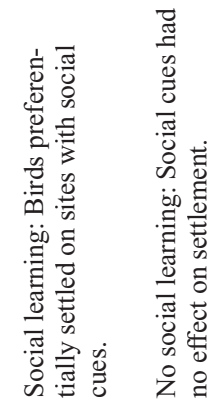

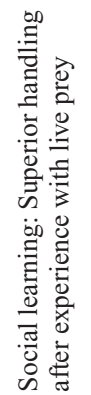
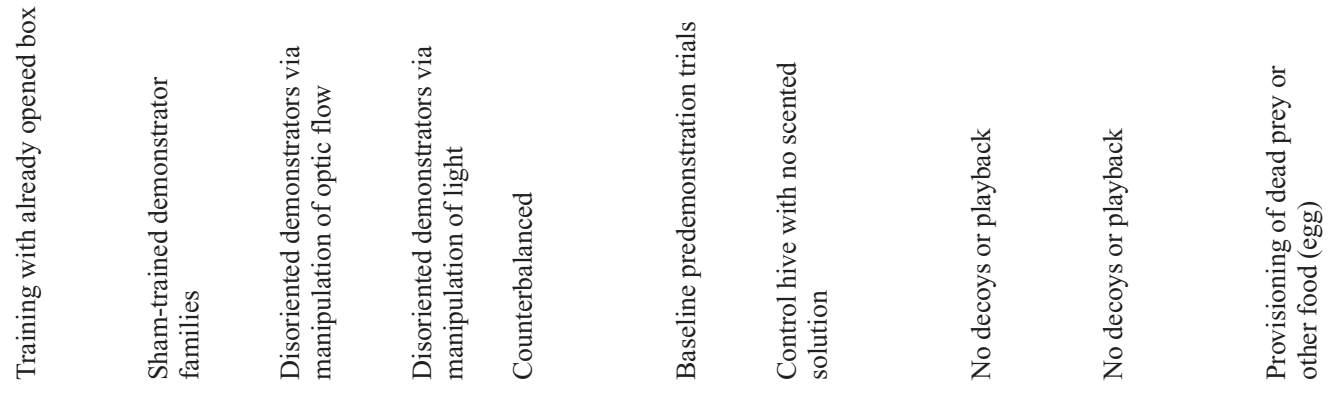

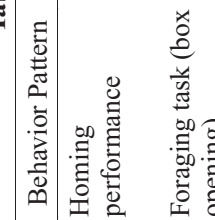

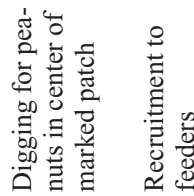

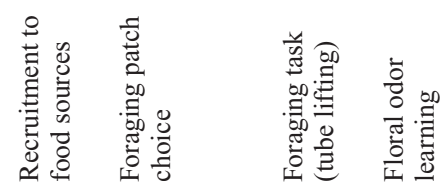

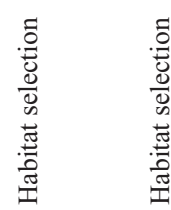

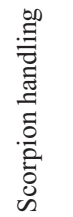

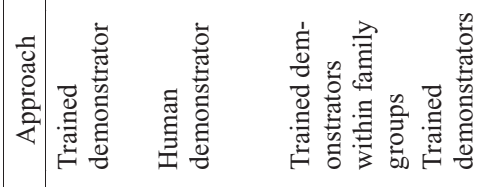

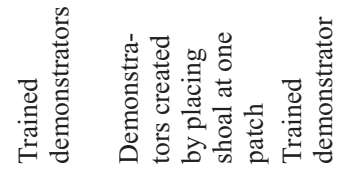

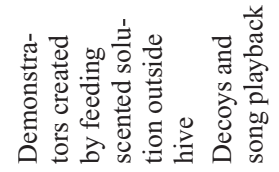

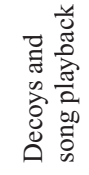

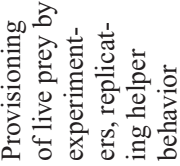

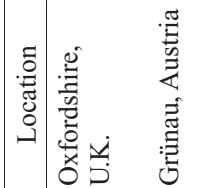

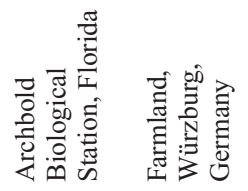

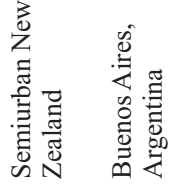

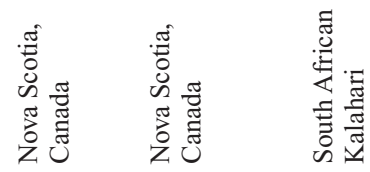

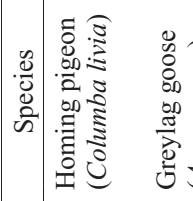

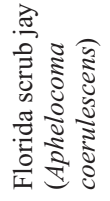

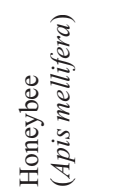

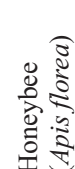

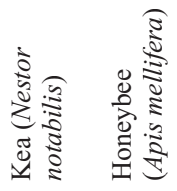

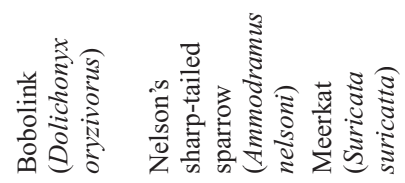

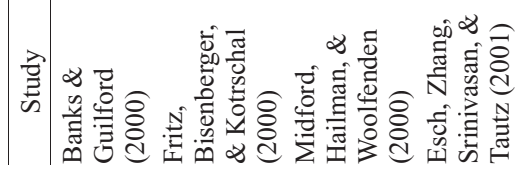

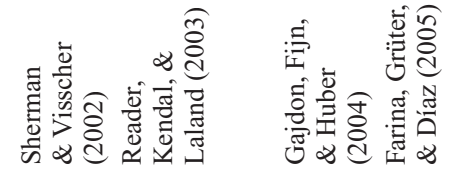

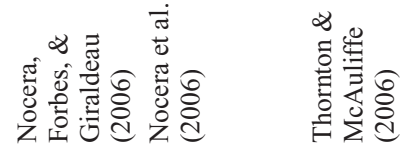




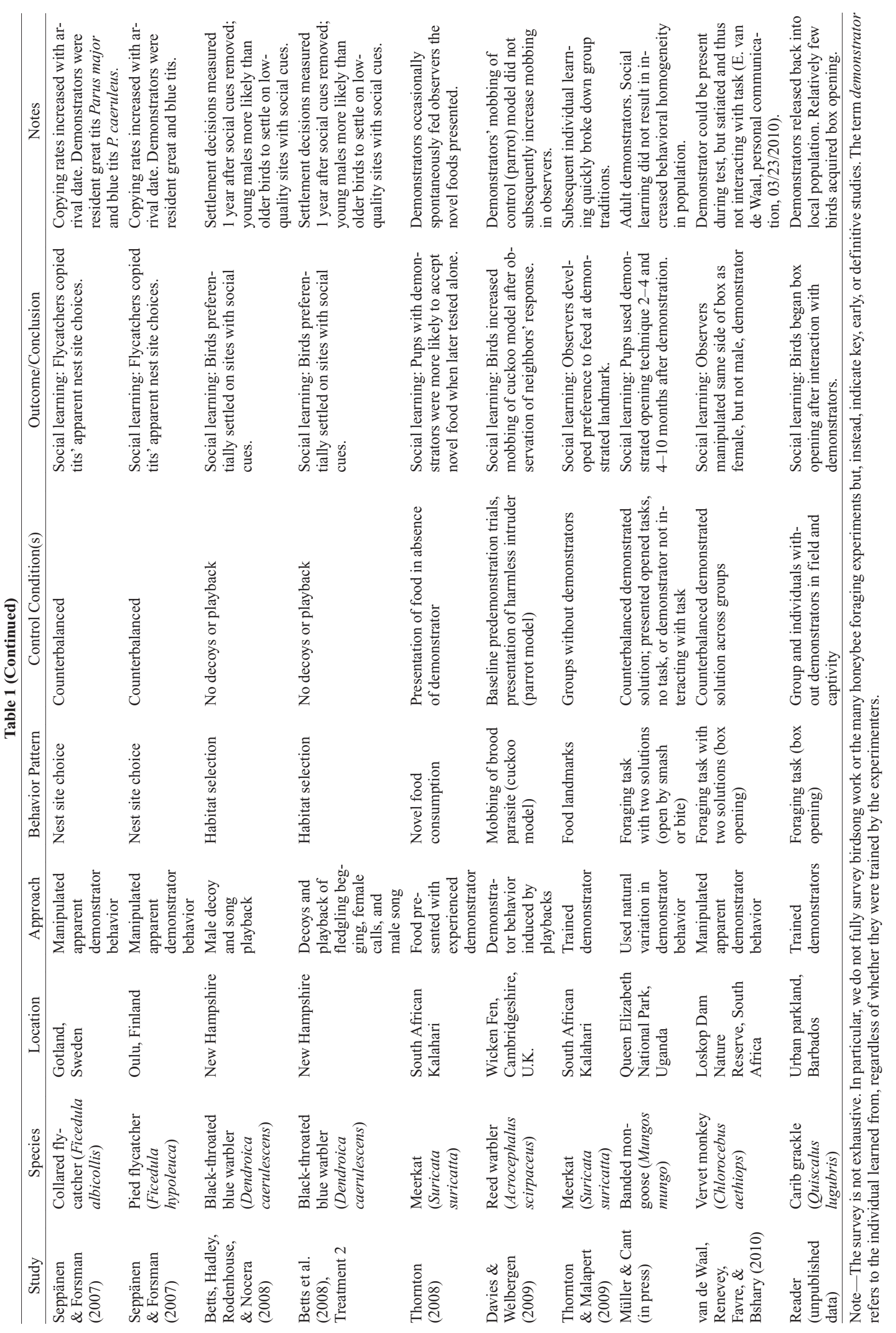


and initially naive young observers, who, in turn, would subsequently become demonstrators for other new recruits to the population.

In a more elaborate manipulation, Warner (1988) replaced entire populations of bluehead wrasse (Thalassoma bifasciatum) in Panama with populations captured on other reefs. The introduced fish discovered and adopted novel mating sites to which they remained faithful for 12 years (four generations). These sites were novel in that they had not been used by the population that had lived there before them. Follow-up studies transplanted the male and female portions of a population separately (Warner, 1990a). Removal of females, but not of males, from a population resulted in the loss of mating sites. Thus, female behavior was responsible for the maintenance of the mating site traditions, a view supported by observations that females arrive together at mating sites.

Warner (1990b) also performed whole-population transplants twice in succession. Mating sites changed after the first transplant. However, and consistent with the hypothesis that transplanted individuals make similar choices about where to mate, sites used after the second transplant were very similar to those used after the first. Warner (1990b) suggested that resource assessment can lead to a predictable pattern of mating site use but that this resource assessment does not occur when a tradition is already in force, which is particularly likely when quality differences between resources are relatively small and costly to assess. In the case of the mating sites, it seems that a surplus of sites is available, and it is more important to be where other individuals are mating than at a slightly superior site where others are not mating. As Warner (1990b) pointed out, when social learning is operating, it may be impossible to predict behavior without knowledge of the prior history of the population. Such experiments, which elegantly demonstrate that local ecology is not fully responsible for gross interpopulation differences in behavior, provide convincing arguments that social learning, rather than environmental constraints, underlie animal traditions that persist over multiple generations.

"Natural" translocations: Interpopulation migrations. The wholesale manipulations of populations (or, effectively, of the environment that they live in), described above in fish, would be problematic for many species and sites, although human introductions of species are numerous for both mammals and birds (Sol, Bacher, Reader, \& Lefebvre, 2008; Sol, Lefebvre, \& Timmermans, 2002) and may provide useful opportunities for social-learning studies (e.g., Jenkins, 1978). Emigrations and immigrations between neighboring populations may also provide parallel data, in the form of natural experiments. However, this approach may be compromised if the behavioral repertoires of the source and destination population are not well known. This is frequently the case because extended research is required to uncover behavioral repertoires and, thus, researchers tend to focus on a particular population. For example, observation of primate groups requires lengthy habituation periods, compromising the following of emigrants from study groups. Moreover, natural experiments are likely to lack the control data needed to firmly establish social learning.

One interesting case study concerns the grooming handclasp, a putative social custom among the chimpanzees (Pan troglodytes) of Mahale, Tanzania (among others) that differs in the details of its performance between different communities (Nakamura \& Uehara, 2004). At Mahale, a female (Gwakulo) changed the way she performed the grooming handclasp after moving between two adjacent communities. After she moved, she adopted the style common in her new community, and individuals in her new group began to perform the style originally preferred by Gwakulo, although only in interactions with her. The grooming handclasp is a product of two interacting individuals, so it remains unknown whether Gwakulo learned the alternative technique after arrival or whether any members of her new group learned the imported technique from her. If future studies document more such examples of intergroup migration followed by changes in behavior of individuals within the recipient group, the case for regional diffusions based on geographic distance (e.g., van Schaik et al., 2003) will be considerably strengthened.

Manipulating the environment: Novel resources or tasks. Less extreme than replacement of the entire habitat for a group of individuals, other forms of manipulating the environment have involved the provision of various resources that are otherwise unavailable. Such provisioning has involved both entirely novel resources and those that conspecifics are utilizing in other locales (Reader, Nover, $\&$ Lefebvre, 2002). The goal of provisioning is to discover whether a behavior is locale specific because of local knowledge or because of differences in local resources. Novel responses to the new resources and any subsequent diffusion of these novel responses through a group can be examined, with particular reference to whether diffusion occurs through individual or social learning.

Matsuzawa and colleagues (Biro et al., 2003; Matsuzawa, 1994; Matsuzawa \& Yamakoshi, 1996) adopted this approach, providing a naturally nut-cracking community of wild chimpanzees at Bossou, Guinea with two species of nuts unavailable at Bossou: the Coula nut, cracked by chimpanzees elsewhere in West Africa, and the Panda nut, cracked by chimpanzees living just $50 \mathrm{~km}$ from Bossou.

Initially, a single individual was observed at Bossou cracking the Coula nuts without hesitation, with other group members showing intense interest in her nutcracking and the majority of the group gradually adopting Coula nut cracking. Panda nuts, on the other hand, were cracked only a handful of times, then completely ignored by all. These observations could be explained by the initial Coula cracker's being a past immigrant to Bossou, from a community where Coula cracking was habitual, and her behavior's influencing the rest of the group to accept Coula nuts. The introduced nuts did not require innovation in tool-using techniques, only adaptation of an existing technique to an unfamiliar target item. Although recent genetic data suggests that the initial Coula nut 
cracker was indeed an immigrant (Shimada et al., 2004), the role of this probably knowledgeable individual in diffusion of the behavior through the Bossou community of chimps remains questionable. Specifically, there is little to suggest that social, rather than individual, learning was responsible for an increasingly large proportion of the Bossou group adapting their existing nut-cracking skills to the processing of a previously unfamiliar nut. That said, the edible Panda nut did not become a similarly attractive item for the chimpanzees. In a sense, the community thus acted as its own control group, providing circumstantial evidence for the influence of a knowledgeable demonstrator in facilitating novel behavior in its observers. However, although the presentation of novel resources or tasks can determine the readiness of a population to utilize them and potentially can exclude social learning as an explanation, proof of social learning is not possible with such a manipulation (but see Kendal, Custance, et al., 2010).

Recently, Gruber, Muller, Strimling, Wrangham, and Zuberbühler (2009) tackled the problems associated with studying a single population, and the difficulty of drawing inferences from a single introduction event with no clear control or comparison, by performing identical manipulations with two communities of chimpanzees living under highly similar ecological conditions but with considerably divergent tool use repertoires (the Sonso and Kanyawara communities of Budongo Forest and Kibale National Park, Uganda). At both sites, Gruber et al. drilled and baited holes in logs with honey, first at depths that chimpanzees could access by hand, then deeper, so that the honey could no longer be reached without a tool. Kanyawara chimpanzees, whose tool repertoire includes use of sticks in extractive foraging, spontaneously used sticks to obtain honey from the deeper hole. Sonso chimpanzees, never observed using sticks for foraging, instead manufactured leaf sponges for the task. Leaf sponging is a "chimpanzee universal" (Whiten et al., 2001): a tool use behavior observed at all chimpanzee field sites (including Kanyawara) so far studied.

This segregation of responses was absolute; not a single Sonso chimpanzee used a stick tool, and not a single Kanyawara chimpanzee used a leaf sponge. The conclusion that the behavior of both communities was scaffolded by their existing knowledge of tools is compelling, although a direct demonstration of social learning being responsible for either (1) maintaining the divergence between the existing tool use repertoires of the two communities or (2) conformity in both communities' responses to the experimental apparatus is lacking (Call \& Tennie, 2009). Nonetheless, Gruber et al.'s (2009) work provides a fascinating glimpse into the possibility that situations involving multiple populations and echoing the highly controlled captive systems examined by Whiten and his colleagues (Whiten, Horner, \& de Waal, 2005; Whiten et al., 2007) can be constructed and examined in the wild.

Manipulating individual behavior: Foraging in fish and mammals. Several field studies, by manipulating the social environment, have focused on the role of key individuals in effecting changes in the behavior of others within their group. Experimental manipulations typically entailed altering the behavior of key individuals from its current state either (1) by explicit training or exposure to specific stimuli or (2) through direct control by experimenters causing key individuals to provide pertinent information to other group members.

The latter design is exemplified by work on Trinidadian guppies (Poecilia reticulata) and vervet monkeys (Chlorocebus aethiops). Reader et al. (2003) examined foraging site choice by guppies in the wild by creating two visually distinct feeding sites of equal quality. One of the sites also contained a small shoal of locally captured fish held within a transparent container, effectively creating a group of demonstrators feeding at one site. Fish preferentially entered the feeding site with the shoal, and, crucially, this preference was maintained in a subsequent test phase, when the demonstrator shoal was no longer present, demonstrating social learning of a feeding site as a result of a tendency to approach feeding conspecifics.

Van de Waal, Renevey, Favre, and Bshary (2010) examined foraging in free-living vervet monkeys, elegantly taking advantage of the fact that dominant individuals monopolized a feeding task to create a single demonstrator in each group without the need for training in isolation. By providing dominant individuals with a feeding task with two solutions (two doors to open a box), but with one solution blocked, observers in different groups viewed only one of the two solutions. Observers were then tested with both solutions available, with observers of female, but not male, demonstrators preferentially exhibiting the demonstrated solution.

In a compelling series of studies, Thornton and colleagues examined the role of social learning in the development of wild meerkats' foraging behavior (Thornton, 2008; Thornton \& McAuliffe, 2006; Thornton \& Raihani, 2010). Initial work focused on helpers (individuals older than 3 months who assist parents in rearing young) teaching prey-handling skills to pups through the provisioning of pups with potentially dangerous but disabled scorpions either with their stings removed or already killed (Thornton \& McAuliffe, 2006). In playback experiments in which the begging calls of pups either younger or older than those present were broadcast from hidden speakers, helpers provided a higher proportion of dead or disabled prey in response to younger pup calls and more intact prey to older pup calls, thus showing sensitivity to the age of the perceived audience (Thornton \& McAuliffe, 2006). Pups given experience of disabled live prey derived the largest benefits in future prey-handling attempts, confirming the benefits of this provisioning.

Meerkat pups were also tested for their acceptance of novel foods by virtue of their association with demonstrators (Thornton, 2008). Pups received unfamiliar food (eggs) with or without an accompanying helper. About half the pups tasted the eggs when a helper was nearby and itself feeding on the food, whereas none did so when alone, and in a subsequent test, in which the same pups were presented with eggs and no helper, only those pups that had previously eaten eggs did so. Pups given two 
consecutive trials with only eggs and no helper did not start feeding on eggs until they were introduced to them by a helper. Taken together, these findings suggest that the experience of feeding on an unfamiliar food together with a knowledgeable conspecific demonstrator significantly sped up pups' acceptance of that food. Similar results were obtained in another set of tests involving scorpions (Thornton, 2008). Data from the mongoose (Mungos mungo) extend on these findings, showing that foraging techniques, as well as food preferences, can be socially acquired and long-lasting (Müller \& Cant, in press). Adults presented with plastic containers filled with food were found to consistently open them one of two ways, by smashing or biting. Later, when dependent pups were present, demonstrators were given containers to open. Pups exhibited the same opening technique as their demonstrator when independent and away from the adult demonstrator, even 10 months later. Control conditions, in which demonstrators either received readyopened containers or no containers, did not result in pups' matching the preferred technique of the demonstrator, thus eliminating the possibility that matching behavior between pups and demonstrators resulted from a tendency for individuals with similar preferred techniques to associate together. Such research is particularly compelling because it mirrors the likely daily experiences of meerkat and mongoose pups and, as such, can inform us of the importance of social learning in the development of young individuals' foraging skills (see also Lonsdorf \& Bonnie, 2010).

Manipulating individual behavior: Habitat selection and mobbing responses in birds. Birds of many species prospect for nesting sites (Doligez, Danchin, \& Clobert, 2002). In a simple and elegant experimental manipulation, Seppänen and Forsman (2007) manipulated the apparent nest site choices of resident great and blue tits before the arrival of two species of migratory flycatchers by painting circles on the front of tit nestboxes and triangles on nearby empty nestboxes (or vice versa). As a result, it appeared that all the tits in each study location had chosen to nest in boxes with one of the two arbitrary symbols. Empty nest boxes painted with the same symbols were made available for flycatchers. The late-arriving migrants copied the apparent nest site choices of the resident tits, demonstrating social learning from heterospecifics. Individuals differed in their utilization of the available social information; in this case, late arrivals were more likely than early arrivals to use social information from resident birds. It is not known whether nest site choices in subsequent years were also affected by the manipulation of social information.

Nocera, Forbes, and Giraldeau (2006) addressed social information use in habitat selection by two synchronously breeding bird species. They provided social cues of habitat choice in the form of conspecific decoys and song playback after the breeding season in one year and then measured visits to the study plots in the subsequent year in the absence of social cues. Social learning could thus be assessed. One species, the bobolink (Dolichonyx oryzivorus), demonstrated social learning even to suboptimal habitats, and another, less social species, Nelson's sharp-tailed sparrow (Ammodramus nelsoni), did not. A related study of black-throated blue warblers (Dendroica caerulescens, a migrant songbird) addressed the relative role of habitat quality and social information in habitat choice by providing social information in habitats of varied quality (Betts, Hadley, Rodenhouse, \& Nocera, 2008). The effect of social information (decoys and playbacks) was compared with a control condition (silence), with the social information presented one year and settlement decisions assessed the next. Young birds were more likely than older birds to immigrate to low-quality sites because of social information. Again, as in bluehead mating site choice (Warner, 1990b), social information can override the available "ecological" information, at least for a subset of the population. An observational study of the warblers showed that male song in the postbreeding season was a reliable correlate of breeding success, and thus social cues provided a useful index for habitat quality (Betts et al., 2008). This finding raises the possibility that particular transmission avenues can be deliberately blocked by experimenters to investigate social learning. Moreover, anthropogenic noise (Slabbekoorn \& Ripmeester, 2008) may block such social information, illustrating how anthropogenic disturbance could disrupt social learning.

Another compelling playback study manipulated demonstrator behavior by the playback of mobbing calls, investigating how birds respond to potential brood parasites (Davies \& Welbergen, 2009). Mobbing call playback caused reed warblers (Acrocephalus scirpaceus) to mob cuckoo or parrot models presented to them, while their nesting neighbors could observe. Subsequent tests of model presentations to the neighboring birds found that mobbing responses to the cuckoo, a harmful brood parasite at the study site, were acquired and maintained even 6 days later, whereas mobbing of parrots, not a brood parasite, was not acquired by the neighbors. The finding of differential learned responses to potentially harmful and harmless models is strikingly similar to the findings of studies of the role of predispositions in predator avoidance learning in captive macaques, where avoidance of snakes, but not flowers, is socially transmitted (Mineka \& Cook, 1988). Reed warblers may preferentially learn about cuckoos because of a genetic predisposition or because of previous exposure to cuckoos at the field site (Davies \& Welbergen, 2009). The experiment thus illustrates the important message that social learning, ecology, and genetic predispositions will interact in the formation and finetuning of behavioral responses. Social learning cannot be viewed as a stand-alone method of acquiring behavior that is independent from either genes or environment.

Manipulating individual behavior: Training demonstrators. Perhaps the most prolific of our three subcategories of field experiments incorporates studies that undertake a combination of manipulations of both the environment and individual behavior. Such approaches provide particularly powerful tests of social learning. Manip- 
ulating the environment exposes individuals to problems to be solved (often involving novel tasks or apparatuses, thus increasing the likelihood that the solution needs to be learned), whereas manipulating individuals introduces a salient and specific behavior whose subsequent appearance in others (when demonstrators are removed) can provide compelling evidence for the spread of information by social learning, particularly if compared with the behavior of control groups without demonstrators.

In what is, to our knowledge, the earliest such design, Lefebvre (1986) studied the establishment of a foraging tradition in feral pigeons by training demonstrators in captivity and then releasing them into a group of naive conspecifics. The task, piercing the covers of grain boxes, was acquired more rapidly by members of the group with demonstrators than by members of a control group without demonstrators. The control group did discover box piercing for themselves, but with much greater latency than the group with trained demonstrators, and only following accidental tearing of box covers by birds walking on them when they were rain-soaked (Lefebvre \& Palameta, 1988). Interestingly, a captive group tested on an identical design with a single trained demonstrator experienced delayed diffusion of the behavior, probably because of scrounging by naive individuals. Removal of knowledgeable individuals from the captive group, simulating the emigration of individuals that would occur in open populations, sped the spread of box piercing. A similar test presented to a feral vervet monkey troop in Barbados provided no evidence for social learning. One juvenile male discovered how to open the boxes and subsequently opened boxes while in close proximity to other troop members on over 70 occasions. However, the behavior never spread (Lefebvre \& Palameta, 1988).

Training of demonstrators on a foraging task has also provided evidence of social learning in white-throated magpie jays (Calocitta formosa) and Florida scrub jays (Aphelocoma coerulescens) (Langen, 1996; Midford, Hailman, \& Woolfenden, 2000). Midford et al. trained and tested birds in family groups. The task involved digging for peanuts hidden inside conspicuously marked rings, and training continued until at least one member of the group became proficient. In subsequent seasons, the trained individuals were repeatedly given the opportunity to demonstrate their knowledge in front of newly fledged young. Control family groups that contained no trained individuals had no young acquire the task, whereas juveniles hatched into trained families acquired the behavior from older members of their group and, crucially, performed it not just in their presence (social information use), but also at feeding sites before demonstrators arrived (learning), although unfortunately, the article does not indicate how often juvenile performance was observed before demonstrator arrival. Interestingly, in contrast with findings from pigeons (Giraldeau \& Lefebvre, 1987), the opportunity for juvenile jays to scrounge during demonstrations, increased experimentally by dividing food items into several pieces, appeared to enhance rather than inhibit learning. Furthermore, the social status of the trained in- dividual appeared to affect opportunities to demonstrate; the presence of naive dominants inhibited subordinate models from performing, indicating that the identity of an innovator can have important consequences for the fate of innovations.

Using two artificially created landmarks and trained demonstrators exhibiting a preference for one over the other, Thornton and Malapert (2009) examined the transmission of foraging patch preferences in wild meerkats. Individual demonstrators were trained to associate food rewards with one of two locations marked by distinctive landmarks, and they had subsequent opportunities to perform their choices in front of, and be joined at the landmarks by, naive members of their group. Although untrained individuals initially showed no preferences for one landmark over the other, through repeated demonstrations by both trained conspecifics and, later, untrained individuals who had developed a preference by virtue of having been rewarded in the presence of trained models, a simple tradition of choosing the demonstrated landmark developed in several replicate groups. In two control groups without any trained demonstrators, no such traditions emerged. Interestingly, however, the traditions were relatively short-lived, with preferences for the two landmarks becoming equal within days, probably a result of individual learning about the alternative landmark. The Thornton and Malapert study provides a rare glimpse into both the appearance and the gradual breakdown of group traditions and confirms individual learning as a powerful modifier of socially acquired behavior.

Gajdon, Fijn, and Huber (2004) combined the training of demonstrators in a wild population of keas (Nestor notabilis), a highly social species established to be a proficient social learner in the laboratory (Huber, Rechberger, $\&$ Taborsky, 2001), with presentation of a novel experimental apparatus. Rather than choosing between alternatives, individual keas needed to learn to solve a novel foraging task. A single individual from a wild population was trained out of sight of other birds and was then allowed to perform in view of naive observers. Surprisingly, the trained model did not appear to aid naive observers' learning of the task. Only 3 of 21 naive birds learned to solve the task, and access to demonstrators failed to effect any increase in the frequency with which other birds interacted with the apparatus. The contrast with captive studies of keas, including a pilot experiment with an apparatus identical to that used in the wild, is stark. The performance of trained demonstrators outside of a laboratory setting is often hard to control, a problem encountered repeatedly in work bridging the gap between experiments with captive and wild animals (e.g., Drea \& Wallen, 1999; Langen, 1996; Nicol \& Pope, 1994). In the Gajdon et al. study, the trained kea demonstrator and most naive keas were prevented from interacting with the apparatus by a dominant individual who had learned (possibly by having observed the demonstrator) to solve the task. Such problems could be solved by designing the apparatus so that the demonstrator can manipulate it without disturbance-for example, by either allowing only the demonstrator access to the 
apparatus or placing the demonstrator within a transparent enclosure - although such methods carry the disadvantage that demonstrators and observers cannot interact together with the apparatus. Such a methodology has been used by one of the authors (S.M.R.) to move captive-trained birds from site to site and to provide multiple demonstrations at each field site by sequentially revealing tasks for the demonstrator.

Although foraging-related tasks constitute the most common forms of demonstrator training in studies of social learning, training demonstrators in other domains provides interesting parallel designs with similar ecological relevance. For example, Banks and Guilford (2000) trained homing pigeons to navigate back to their home loft through repeated releases from a distant site. Following training, during which the birds gradually improved in their ability to home, each demonstrator was released with a single naive conspecific and was allowed to demonstrate the homing route once. During paired flight with a knowledgeable partner, the performance of inexperienced birds exceeded that of fully naive control pairs (confirming that they were, indeed, receiving an accurate demonstration of the homing route). However, on a subsequent solo release, the tutored birds homed no faster than control birds, indicating that experiencing the performance of a knowledgeable navigator does not aid navigational learning, possibly due to a form of overshadowing between social and navigational cues, producing a scrounging-like passenger/ driver effect (Burt de Perera \& Guilford, 1999). Nonetheless, it is possible that repeated demonstrations or larger demonstrator groups would enhance the social learning of travel routes. The Banks and Guilford study has obvious relevance to any species whose members travel in groups and is particularly innovative, since animal movement patterns present many fascinating questions regarding potential traditions. For example, little is currently known about the precise role of social (and individual) learning, relative to genetically encoded direction preferences (Helbig, 1991; Mukhin, Kosarev, \& Ktitorov, 2005; Plotkin, Byles, Rostal, \& Owens, 1995) in the establishment, maintenance, and fine-tuning of migration routes in annual long-distance migrants that travel in mixed-age, mixed-sex, and mixed-experience groups. The fact that novel migration routes can evolve rapidly (Berthold, Helbig, Mohr, \& Querner, 1992) reinforces the message that genetic adaptations may evolve quickly and should not be excluded as an explanation for the appearance of novel behavior patterns.

Finally, in populations in which the temporary isolation, training, and reintroduction of designated models into a group of naive conspecifics is not feasible, fortuitous events can simulate the appearance of trained demonstrators. Hannah and McGrew (1987) studied semicaptive chimpanzees in Liberia, who were released, one by one, from a sanctuary into an increasingly self-sufficient (although still provisioned) existence on a 10-ha island. Within hours of release, Samantha, the 11th individual released, was cracking oil-palm nuts with a stone hammer, a behavior never previously observed in any of the other individuals. Within a month, 9 of the 13 other individuals released on the island were also cracking nuts, some with the aid of materials introduced onto the island by researchers following the initial observations of nut cracking. Although there was no equivalent, matched group without a potential demonstrator, the evidence is suggestive of a key role for a single demonstrator in the diffusion of the behavior. However, the speed with which nut cracking spread through the population contrasts with the lengthy developmental time course of chimpanzees' toolusing skills in general (Inoue-Nakamura \& Matsuzawa, 1997; Lonsdorf, Eberly, \& Pusey, 2004; see Lonsdorf \& Bonnie, 2010). Nut-cracking individuals thus may not have acquired nut cracking from Samantha. Hannah and McGrew speculated that, instead, they may already have known how to nut crack and simply had "their memories prompted by her actions" (p. 43).

Honeybee social learning of food locations and odors. Honeybees socially learn from conspecifics within the hive. We cannot fully review the many published studies here or in Table 1 but do point to experiments that illustrate a variety of valuable approaches to field sociallearning experiments. For example, demonstrators can be created by feeding them scented foods (Farina, Grüter, \& Díaz, 2005) or training them to particular locations (Gould, 1975b). Social cues such as the waggle dance can be modified or eliminated in many ways - for example, by manipulations of the optical or light environment (Esch, Zhang, Srinivasan, \& Tautz, 2001; Sherman \& Visscher, 2002). Moreover, data on the dance of honeybees have led to the construction of an effective mechanical demonstrator that can be precisely controlled by experimenters and direct observers to particular locations (Michelsen, Andersen, Storm, Kirchner, \& Lindaner, 1992).

The controversy over the interpretation of honeybee waggle dance studies also illustrates the general need to eliminate from social-learning experiments the confounding effects of residual cues left by demonstrators, demonstrator activity in the presence of subjects, and environmental factors (Gould, 1975b, 1976). Moreover, waggle dance studies illustrate how conflicting data can result because social information use can vary between individuals and conditions (Grüter \& Farina, 2009; Sherman $\&$ Visscher, 2002). The techniques utilized in the study of honeybee social learning could be adapted to other animals, and the increasing number of studies uncovering social learning or social information use in invertebrate species that are relatively easily manipulated in field settings (Laidre, 2010; Leadbeater \& Chittka, 2007) suggest that invertebrates will provide much useful information on the ecology of social learning.

\section{Bringing Behavior Into the Laboratory: Laboratory Experiments Based on Field Observations}

All laboratory studies of social learning will, to a greater or lesser degree, focus on the typical behavioral repertoire of the species under study. Several studies, however, have directly taken field observations and 
brought them into the laboratory, even explaining how social learning can facilitate invasion of a new habitat (Terkel, 1996). For example, demonstration of the time and energetic savings for squirrels opening nuts and rats extracting seeds from pine cones with exposure to a trained model illustrate how ecologically relevant laboratory studies can address the function of social learning (Terkel, 1996; Weigl \& Hanson, 1980). We discuss some instructive examples of laboratory experiments undertaken explicitly to uncover the mechanisms or functions of acquisition of specific behavior patterns observed in the field. Some demonstrate the necessity of social information for acquisition, whereas others show that social information is not required.

Rats diving for food. Wild rat colonies along the Po river in Italy dive for and feed on mollusks on the river bed, whereas neighboring colonies, where mollusks are also available, do not (Galef, 1980). This patchy pattern could, like regional differences in chimpanzee behavior (Whiten et al., 1999), be explained by a combination of rare individual innovation and social learning within a locality. However, laboratory experiments demonstrated that social learning is not essential for the development of diving behavior (Galef, 1980). Rats were trained to dive for submerged chocolates (a preferred food) by gradually submerging the chocolates in water. Interaction with trained rats did not lead to diving by naive subjects, but rats trained to swim would dive for food, raising the possibility that diving could result from the social learning of swimming. Social interaction accelerated acquisition of swimming in a domestic rat strain, but experiments demonstrated that swimming could develop before adulthood with or without a trained demonstrator, meaning that social learning was not necessary for the development of swimming and, consequently, the development of diving. In a final experiment, rats that regularly dove to retrieve chocolate were shown to stop diving for chocolate if other food was made available, even though chocolate was the preferred food. Galef (1980) suggested that natural shaping, via mollusks being revealed by fluctuations in the water level, could explain the presence of the behavior pattern in at least some wild groups where such fluctuations in water level exist, whereas the availability of alternative food resources could explain its absence in neighboring groups. Moreover, these results suggest further field experiments (Galef, 1980). For example, alternative food resources could be provided to diving populations, with the prediction that diving would cease, whereas nondiving rats could be trapped and tested in the laboratory to determine whether they do not dive because of the availability of other resources.

Primate potato washing. Japanese macaques' (Macaca fuscata) washing of sandy food provides a key, although controversial, example of the spread of a novel foraging behavior that some assert is a result of social learning (Hirata, Watanabe, \& Kawai, 2001; Kawai, 1965). However, experiments providing sandy food to small captive groups of tufted capuchin monkeys (Cebus apella) and crab-eating macaques (Macaca fascicularis) showed that the majority of individuals consistently washed sandy food, without evidence of copying (Visalberghi \& Fragaszy, 1990). Thus, individual discovery could explain the high frequency of food washing in monkeys, including Japanese macaques. Similarly, a study of grackles dunking food in water, also a proposed example of social learning, revealed that under ideal conditions in captivity, many individuals dunk food, even though the behavior is infrequent in the wild (Morand-Ferron, Lefebvre, Reader, Sol, \& Elvin, 2004).

Avian tool use. Observations of tool use and manufacture in woodpecker finches (Cactospiza pallida) and New Caledonian crows (Corvus moneduloides) led to both considerable interest in the method of acquisition of the behavior and speculation that social learning may be involved (Hunt, 1996; Hunt \& Gray, 2004; Kenward, Rutz, Weir, \& Kacelnik, 2006; Kenward, Weir, Rutz, \& Kacelnik, 2005; Orenstein, 1972; Tebbich, Taborsky, Fessl, \& Blomqvist, 2001). However, studies on captive-reared individuals confirmed that social demonstrations of tool manufacture and use were not necessary for tool use to develop (Kenward et al., 2006; Kenward et al., 2005; Tebbich et al., 2001). Such studies illustrate the value of captive studies, in which the prior histories of animals are known and controlled, for understanding processes involved in behavioral development in the wild.

British titmice opening milk bottles. The spread across the United Kingdom of birds opening milk bottles and drinking the cream from the milk surface is often regarded as a classic case of social transmission, although the original investigators were cautious in their interpretation of the process of diffusion (Fisher \& Hinde, 1949; Hinde \& Fisher, 1951, 1972). Milk bottle opening is certainly the best characterized, and perhaps best known, example of the spread of a novel behavior pattern in nonhuman animals. Over 30 years, ornithologists noted the repeated appearance of birds opening milk bottles at tens of sites and in thousands of birds, although the specific opening technique was variable, even within individuals (Fisher \& Hinde, 1949; Hinde \& Fisher, 1972).

Captive experiments on titmice and black-capped chickadees (Poecile atricapilla) cast doubt on social learning as the sole explanation for the spread of the behavior, demonstrating that the rate of spontaneous opening of milk bottles can be quite high, even in seminatural conditions with animals living together and alternative foods available (Kothbauer-Hellman, 1990; Sherry \& Galef, 1984, 1990). The nonsocial origin of milk bottle opening is supported by analyses of the geographical spread of the behavior, which suggested many independent origins (Lefebvre, 1995). However, in chickadees, the rate of learning to open tubs of cream was increased in birds that encountered open food sources (Sherry \& Galef, 1984, 1990), demonstrating that at least one form of social learning can play a role in its spread. The most likely explanation for the spread of bottle-opening behavior was local innovation, where milk bottles were introduced, followed by social learning from bird to bird within that locality (Ingram, 1998). 
Rat food preference learning. Field observations that wild rat (Rattus norvegicus) populations rapidly learn to avoid poisoned bait prompted Galef and colleagues to embark on an extensive and compelling set of laboratory studies on food preference learning, now also the subject of considerable neuroendocrine research (reviewed in, e.g., Choleris, Clipperton-Allen, Phan, \& Kavaliers, 2009; Galef, 2002, 2004), These studies revealed that food preferences could be acquired from other individuals by multiple routes, such as pups' learning from the flavor of their mother's milk, by the scent of conspecific breath, by following others to feeding sites, and by preferring sites marked with excretory cues. When combined with a tendency to avoid novel foods, these socially acquired preferences can allow rats to effectively avoid lethal toxins (Noble, Todd, \& Tucit, 2001). Field studies of food preference learning would be feasible, but to our knowledge, the definitive experiments have not yet been done. However, studies of wild rats in a large outdoor enclosure created demonstrators by adding cinnamon scent to the head region of marked rats and then presented the colony with feeders containing cinnamon- or peppermintflavored food (Berdoy, 1994). Cinnamon-flavored food was preferentially consumed, and a control group without demonstrators had no preference for cinnamon- over peppermint-flavored food. Moreover, the demonstrators did not initially eat cinnamon. Thus, with this elegant design, Berdoy was able to introduce demonstrators while excluding the possibility that subjects' cinnamon preference was the result of simultaneous feeding alongside demonstrators.

Birdsong learning. Birdsong is perhaps the best studied example of social learning, with questions of mechanisms, development, adaptive function, and evolution all well represented. We cannot do justice to this vast field here but note some examples where the methodology of song learning could be adopted to study other domains of social learning. Field studies, such as Jenkins (1978), have established that song learning occurs in the field by examining the song match between birds, the distribution of song groups across a habitat, and changes in songs over time, while ruling out a role for genetic inheritance from the father in song production. Moreover, because birds were translocated to a new habitat as part of a reintroduction effort and the same songs were found in the old and new habitats, it is unlikely that birds living in close proximity and sharing songs had individually adapted their songs to a particular locale (Jenkins, 1978).

Both because song is complex and highly variable and contains arbitrary elements and because laboratory studies have demonstrated that birds copy their tutors, spread of songs through populations can be monitored without intrusive experimental tests of social learning (Beecher, 2008; Lynch \& Baker, 1994; Slater, 1986). This is a major advantage to social-learning studies based on the vocalizations of both birds and cetaceans. It remains an open question whether other behavior patterns exist that have the characteristics of song that allow social learning to be demonstrated on the basis of the match between the be- havior of two or more individuals, although it has been argued that, like bird song, some primate foraging behaviors are complex and highly variable and contain arbitrary elements (Byrne, 2007). However, where acts are rewarded, a match in the behaviors of individuals will not be informative, because animals are likely to independently arrive at similar ways of doing things. Consequently, acquisition of arbitrary acts, such as the choice between two equally rewarding foraging sites (Thornton \& Malapert, 2009; Warner, 1990b) or group-specific arbitrary social customs (Nakamura \& Uehara, 2004), can be particularly useful in elucidating social learning.

Birdsong studies illustrate several relevant approaches for social-learning research. For example, telemetry can examine what kind of information animals attend to in the field and, hence, can establish likely transmission routes. For example, juvenile song sparrows prefer to "eavesdrop" on song interactions between adults, rather than solo songs, emphasizing how social interactions are important to song learning (Beecher, 2008; C. N. Templeton, Akçay, Campbell, \& Beecher, 2010). Playback studies that compare birds' responses to known and unknown songs can be used to determine what birds know (Stoddard, Beecher, Homing, \& Campbell, 1991). Similar methods could be adopted to other behavior patterns: Perhaps animals preferentially attend to novel foraging acts, whereas they ignore acts already in their repertoire, or perceive as more salient behaviors occurring in specific social contexts. Birdsong researchers have also successfully combined field and captive studies, controlling the access of individuals to potential demonstrators (e.g., Nordby, Campbell, Burt, \& Beecher, 2000; Zann, 1990). For example, Zann captured juvenile finches of different ages and placed them in an enclosure within the source colony, thus precluding close interaction with their fathers. The younger the birds were when they were confined, the less their songs matched those of their fathers, establishing the importance of interaction with the father during the sensitive period for learning his song. Birdsong studies illustrate how the timing, context, and source of social information are important determinants of social learning.

\section{Discussion}

Social learning has been experimentally demonstrated in the wild in a range of taxa and a range of contexts. Our survey is probably incomplete but, nonetheless, includes learning of foraging resources, locations, and techniques; responses to predators and brood parasites; travel routes; mating sites; and habitat and nest site choices. Moreover, socially acquired responses have been shown to be longlasting in wild populations (e.g., Betts et al., 2008; Müller \& Cant, in press; Warner, 1988). Social learning is thus both widespread and relevant to animal ecology, and we would argue that, as with many other aspects of ecology, social learning is beneficially studied in situ. Not only are field experiments feasible, they are also a vital part of understanding social learning. Although the number of field experiments has expanded dramatically in recent 
years, such studies remain relatively rare. Moreover, studies of captive animals (e.g., Kenward et al., 2005; Tebbich et al., 2001) have shown that behavior patterns originally ascribed to social learning may not require social information for their development, which provides a note of caution when population differences in behavior can perhaps be too readily labeled as cultural phenomena without sufficient evidence. That said, the fact that a behavior can develop in the laboratory without social learning does not rule out a role for social learning of that behavior in natural circumstances (Kenward et al., 2006).

Numerous approaches have been taken to study social learning in the field, ranging from manipulations of entire populations to insertion into populations of trained individuals or novel resources, the blocking of transmission avenues, or creating the appearance that animals are performing a particular behavior or choosing one resource over another. Studies of social or public information use could be relatively simply extended to also examine social learning by subsequently testing individuals without demonstrators present; we noted many such missed opportunities in the published literature. Where control populations are not available, baseline measures can be taken or multiple successive tests with altered task demands can be administered, although such "controls" would be less powerful than concurrently tested control groups.

Both field and captive studies contribute to a fuller understanding of the processes of socially mediated learning, but are field studies really essential? Our survey illustrates that they are. Field work has provided evidence for processes not yet demonstrated in the laboratory, such as social learning of brood parasite mobbing or mating sites (Davies \& Welbergen, 2009; Warner, 1988), and has produced results contrary to those of laboratory studies (e.g., Gajdon et al., 2004; Lefebvre, 1986). Thus, in some cases, field studies may be more feasible than captive studies and may open new avenues for research in the laboratory, as well as demonstrating that social learning is relevant to the life of wild animals. Rigorous field demonstrations of social learning, its underlying processes, and its adaptive consequences are feasible and essential.

Closing the gap between the laboratory and the wild. Galef and Allen (1995) highlighted how the almost exclusive use of observer-demonstrator pairs in sociallearning research, although providing a simple and powerful design for elucidating transmission mechanisms, suffers from being largely unrepresentative of the learning opportunities that animals encounter in the wild. Social animals typically have access to the behavior of multiple potential demonstrators and, outside the laboratory, are also likely to be exposed to a variety of potential resources and techniques for obtaining them. What effect rich physical and social environments have on the (1) emergence of novel behaviors, (2) dynamics of their diffusion among group members, (3) multigenerational survival of traditions, and (4) transfer of knowledge between groups are questions that clearly require a move away from observerdemonstrator pairs toward more ecologically relevant designs, even in captivity (Laland, Richerson, \& Boyd, 1993; Whiten \& Mesoudi, 2008).
With these criticisms in mind, there has been burgeoning interest in creating captive methodologies that better reflect conditions in the wild. Transmission chain designs (e.g., Curio, Ernst, \& Vieth, 1978; Galef \& Allen, 1995; Laland \& Plotkin, 1992; Laland \& Williams, 1997) in which trained demonstrators pass on behavior patterns to observers, who then become demonstrators for the next observers in the chain, are useful for examining the fidelity of transmission and, thus, the potential longevity of traditions. Transmission chains simulate the addition of naive individuals to groups. So-called open group diffusion studies again provide a more naturalistic design than do more rigorously controlled but simpler captive studies (Whiten \& Mesoudi, 2008). Diffusion studies replicate situations in which novel behavior patterns are introduced through either innovation or arrival of migrants: Inventions must spread from a single demonstrator through a group, potentially competing with alternative solutions that emerge and spread in the meantime. The fates of such alternative solutions are interesting in themselves and can help determine how the identities of independent innovators influence the transmission versus extinction of behavioral alternatives. Indeed, the innovations of subordinate individuals may represent dead ends in terms of transmission (Bonnie, Horner, Whiten, \& de Waal, 2007; Laland \& Reader, 1999; Midford et al., 2000; Reader \& Laland, 2001), due either to the lack of opportunity of subordinates to perform a behavior (if the resource is limited) or to the low salience of their demonstrations (their behavior is not attended to by fellow group members). Diffusion studies with chimpanzees typically train a high-ranking individual as a demonstrator to reduce such problems (e.g., Whiten et al., 2005). Diffusion studies provide the opportunity to study complex transmission dynamics and are likely to proliferate as an informative, powerful, and ecologically relevant captive design.

Open questions of social learning. Field experiments raise new research questions and also point to routes to address open questions concerning social learning. Field experiments are particularly valuable in addressing the adaptive significance of social learning but can assist in establishing the generality of laboratory findings concerning all four of Tinbergen's (1963) questions: mechanism, development, adaptive function, and evolution. Although much has been learned through both laboratory and field studies regarding mechanism and function, developmental and evolutionary perspectives remain relatively unexplored. For example, are there sensitive periods for social learning or developmental changes in who and what aspects of behavior individuals attend to? Do individuals specialize in learning individually or socially, and, if so, how does development shape specialization? What selective pressures, if any, drive some species to evolve increased sensitivities to socially acquired information, and what can we learn from identifying informative cases of convergence? Moreover, field experiments can address issues that do not fit neatly into Tinbergen's scheme, such as the evolutionary consequences of socially acquired traits or the role of social learning in niche construction (Odling-Smee, Laland, \& Feldman, 2003; Wyles, Kunkel, \& Wilson, 1983). 
An obvious question is the taxonomic distribution of social learning. The majority of the studies ( 12 of 23 studies, 12 of 20 species $^{4}$ ) in our survey (see Table 1) focused on birds, with fish, social insects, and mammals also represented. Surprisingly, and despite the intense interest in primate social learning (Fragaszy \& Perry, 2003), social learning in wild populations of nonhuman primates has been definitively demonstrated experimentally only very recently, and in a single study (van de Waal et al., 2010). Social learning is taxonomically widespread, although whether the same processes are involved in the various instances reported is currently unknown.

The observed taxonomic distribution of animals utilizing social learning will reflect research traditions and the ease of performing field experiments with different taxa, and it remains an open question to what extent ecology versus other species characteristics, such as cognitive ability or social structure, determines species differences in social learning (Lefebvre \& Giraldeau, 1996; Lefebvre \& Palameta, 1988; Reader \& Laland, 2002; Reader \& Lefebvre, 2001; J. J. Templeton, Kamil, \& Balda, 1999). For example, will social learning be more widespread, faster, or involve different transmission mechanisms among egalitarian species or those with well-defined hierarchical organization (see Kendal, Custance, et al., 2010; Lonsdorf \& Bonnie, 2010)? Is the capacity to learn socially an adaptive specialization, or does much social learning result from the by-products of other behavior, such as grouping (van der Post \& Hogeweg, 2006)? For example, differences in the social learning of escape routes between wild guppies, domesticated zebrafish (Danio rerio), and domesticated guppies may reflect differences in grouping tendencies (Brown \& Laland, 2002; Lindeyer \& Reader, 2010; Reader et al., 2003). Similarly, local foraging conditions can result in learned preferences for particular conspecific versus heterospecific tutors (Carlier \& Lefebvre, 1997; Dolman, Templeton, \& Lefebvre, 1996). The extent to which social-learning propensities and preferences to attend to particular demonstrators vary across individuals or are shaped by experience or local conditions in the field is unknown (Kendal, Coolen, van Bergen, \& Laland, 2005; Laland, 2004; Leadbeater \& Chittka, 2009; Toelch et al., 2009). Indeed, the presence or absence of social learning may not be a species characteristic. Instead, the use of social information may vary across contexts and ecologies, and discovery of novel forms of social learning may be more a matter of designing intelligent experiments than of studying intelligent animals.

Field experiments can also go beyond the issue of whether social information is used to acquire a behavior pattern. For example, several studies have examined the integration of social, individual, and ecological informationnotably, work on habitat selection in birds (e.g., Seppänen \& Forsman, 2007). The integration of different sources of information, including different sources of social information, is an important issue. For example, the relative utility of a given foraging behavior could be influenced by the availability of other foods, the ease of identifying alternate foods, differences in need (e.g., disease promoting peculiar nutritional requirements), local predation risk, cognitive or other constraints on performance of alternative foraging behaviors, or the activities of others (Dewar, 2004; Reader, 2004). A focus on social learning can lead to neglect of other processes involved in the maintenance of traditions. Traditions depend not just on social acquisition of behavior, but also on its retention; if individuals modify socially acquire behavior or innovate, a tradition is lost (Heyes, 1993). Manipulations of the social environment could help determine the role of both demonstrators and nondemonstrators in the spread of behavior, whereas manipulations of the physical environment could address hypotheses regarding the evolution or function of social learning and the maintenance of traditions.

Finally, field experiments can also address applied issues. For example, it has been suggested that social learning provides a way to increase the success of captivereared individuals released into the wild, important for both conservation and farming (Griffin, 2004; Suboski $\&$ Templeton, 1989). One year after release into the wild, captive-reared black-tailed prairie dogs trained to predators in the presence of experienced adults had almost double the survivorship of juveniles trained without experienced adults (Shier \& Owings, 2007), demonstrating that social information can provide a survival advantage, potentially improving conservation programs. Social learning can also be used to train individuals to respond to unfamiliar threats (Griffin, 2004), and manipulation of social cues may therefore facilitate the relocation of problem or impacted species. Under certain conditions, the use of social cues may have maladaptive consequences-for example, when environmental change outpaces traditional behavior. Lastly, local traditions may themselves be the subject of conservation efforts when extinction of a local population destroys local traditions, as well as the individuals exhibiting them (Corten, 2002; van Schaik, 2002; Whitehead, 2010).

\section{AUTHOR NOTE}

We thank L. Lefebvre, B. G. Galef, S. E. Hewlett, and O. Todorov for helpful comments, and T. A. Langen and E. van de Waal for additional information on published studies. S.M.R. is partially funded by a Utrecht University High Potentials grant and thanks the Netherlands Organisation for Scientific Research (NWO) Evolution and Behaviour Programme for additional funding, and McGill University for hospitality during a sabbatical visit. D.B. was supported by a Royal Society University Research Fellowship and by Somerville College, Oxford. Correspondence concerning this article should be addressed to S. M. Reader, Behavioural Biology, Utrecht University, Padualaan 8, P.O. Box 80086, 3508 TB Utrecht, The Netherlands (e-mail: s.m.reader@uu.nl), or D. Biro, Department of Zoology, University of Oxford, South Parks Road, Oxford OX1 3PS, England (e-mail: dora.biro@zoo.ox.ac.uk).

\section{REFERENCES}

BAnKs, A. N., \& GUILFORD, T. (2000). Accurate route demonstration by experienced homing pigeons does not improve subsequent homing performance in naive conspecifics. Proceedings of the Royal Society $B, \mathbf{2 6 7}, 2301-2306$.

BeEcher, M. D. (2008). Function and mechanisms of song learning in song sparrows. Advances in the Study of Behavior, 38, 167-225.

Berdoy, M. (1994). Making decisions in the wild: Constraints, conflicts and communication in foraging rats. In B. G. Galef, Jr., M. Mainardi, 
\& P. Valsecchi (Eds.), Behavioural aspects of feeding (pp. 289-313). Chur, Switzerland: Harwood.

Berthold, P., Helbig, A. J., Mohr, G., \& Querner, U. (1992). Rapid microevolution of migratory behaviour in a wild bird species. Nature, $360,668-670$.

Betts, M. G., Hadley, A. S., Rodenhouse, N., \& Nocera, J. J. (2008). Social information trumps vegetation structure in breeding-site selection by a migrant songbird. Proceedings of the Royal Society B, 275, 2257-2263.

Biro, D., Inoue-Nakamura, N., Tonooka, R., Yamakoshi, G., Sousa, C., \& MatsuzaWa, T. (2003). Cultural innovation and transmission of tool use in wild chimpanzees: Evidence from field experiments. Animal Cognition, 6, 213-223.

Bonnie, K. E., Horner, V., Whiten, A., \& de WaAl, F. B. M. (2007). Spread of arbitrary customs among chimpanzees: A controlled experiment. Proceedings of the Royal Society B, 274, 367-372.

Boyd, R., \& Richerson, P. J. (1985). Culture and the evolutionary process. Chicago: University of Chicago Press.

Brown, C., \& LaLAND, K. N. (2002). Social learning of a novel avoidance task in the guppy: Conformity and social release. Animal Behaviour, 64, 41-47.

Burt de Perera, T., \& Guilford, T. (1999). The social transmission of spatial information in homing pigeons. Animal Behaviour, 57, 715719.

BYRNE, R. W. (2007). Culture in great apes: Using intricate complexity in feeding skills to trace the evolutionary origin of human technical prowess. Philosophical Transactions of the Royal Society B, 362, 577-585.

Call, J., \& Tennie, C. (2009). Animal culture: Chimpanzee table manners? Current Biology, 19, R983-R985.

CARLier, P., \& Lefebvre, L. (1997). Ecological differences in social learning between adjacent, mixing populations of zenaida doves. Ethology, 103, 772-784

Choleris, E., Clipperton-Allen, A. E., Phan, A., \& Kavaliers, M. (2009). Neuroendocrinology of social information processing in rats and mice. Frontiers in Neuroendocrinology, 30, 442-459.

Conover, M. R., \& Perito, J. J. (1981). Response of starlings to distress calls and predator models holding conspecific prey. Zeitschrift für Tierpsychologie, 57, 163-172.

CORTEN, A. (2002). The role of "conservatism" in herring migrations. Reviews in Fish Biology \& Fisheries, 11, 339-361.

Curio, E., ERnst, U., \& VieTh, W. (1978). The adaptive significance of avian mobbing: II. Cultural transmission of enemy recognition in blackbirds: Effectiveness and some constraints. Zietschrift für Tierpsychologie, 42, 184-202.

CURio, E., Ulrich, E., \& Vieth, W. (1978). Cultural transmission of enemy recognition: One function of avian mobbing. Science, 202, 899-901.

Cuthill, I. (1991). Field experiments in animal behaviour: Methods and ethics. Animal Behaviour, 42, 1007-1014.

Davies, N. B., \& Welbergen, J. A. (2009). Social transmission of a host defense against cuckoo parasitism. Science, 324, 1318-1320.

DAWKINs, M. S. (2007). Observing animal behaviour: Design and analysis of quantitative data. Oxford: Oxford University Press.

DEWAR, G. (2004). Social and asocial cues about new food: Cue reliability influences intake in rats. Learning \& Behavior, 32, 82-89.

Doligez, B., Danchin, E., \& Clobert, J. (2002). Public information and breeding habitat selection in a wild bird population. Science, 297, $1168-1170$

Dolman, C. S., Templeton, J., \& Lefebvre, L. (1996). Mode of foraging competition is related to tutor preference in Zenaida aurita. Journal of Comparative Psychology, 110, 45-54.

Drea, C. M., \& Wallen, K. (1999). Low-status monkeys "play dumb" when learning in mixed social groups. Proceedings of the National Academy of Sciences, 96, 12965-12969.

Esch, H. E., Zhang, S., Srinivasan, M. V., \& Tautz, J. (2001). Honeybee dances communicate distances measured by optic flow. Nature, 411, 581-583.

Farina, W. M., Grüter, C., \& Díaz, P. C. (2005). Social learning of floral odours inside the honeybee hive. Proceedings of the Royal Society $B, 272,1923-1928$.

FisheR, J., \& Hinde, R. A. (1949). The opening of milk bottles by birds. British Birds, 42, 347-357.
Fragaszy, D. M., \& Perry, S. (Eds.) (2003). The biology of traditions. Models and evidence. Cambridge: Cambridge University Press.

Franz, M., \& NunN, C. L. (2009). Network-based diffusion analysis: A new method for detecting social learning. Proceedings of the Royal Society B, 276, 1829-1836.

Fritz, J., Bisenberger, A., \& Kotrschal, K. (2000). Stimulus enhancement in graylag geese: Socially mediated learning of an operant task. Animal Behaviour, 59, 1119-1125.

Gajdon, G. K., FiJn, N., \& Huber, L. (2004). Testing social learning in a wild mountain parrot, the kea (Nestor notabilis). Learning \& Behavior, 32, 62-71.

GALEF, B. G., JR. (1976). Social transmission of acquired behavior: A discussion of tradition and social learning in vertebrates. Advances in the Study of Behavior, 3, 77-100.

GALEF, B. G., JR. (1980). Diving for food: Analysis of a possible case of social learning in wild rats (Rattus norvegicus). Journal of Comparative \& Physiological Psychology, 94, 416-425.

GaLeF, B. G., JR. (1988). Imitation in animals: History, definition, and interpretation of data from the psychological laboratory. In T. Zentall \& B. G. Galef, Jr. (Eds.), Social learning: Psychological and biological perspectives (pp. 3-28). Hillsdale, NJ: Erlbaum.

GALEF, B. G., JR. (2002). Social learning of food preferences in rodents: A rapidly learned appetitive behavior. Current Protocols in Neuroscience, 8.5, D1-D8

GALEF, B. G., JR. (2004). Approaches to the study of traditional behaviors of free-living animals. Learning \& Behavior, 32, 53-61.

Galef, B. G., JR., \& Allen, C. (1995). A new model system for studying behavioural traditions in animals. Animal Behaviour, 50, 705-717.

Galef, B. G., JR., \& LaLAND, K. N. (2005). Social learning in animals: Empirical studies and theoretical models. BioScience, 55, 489-499.

GiRALDEAU, L. A., \& LeFeBVRe, L. (1987). Scrounging prevents cultural transmission of food-finding behaviour in pigeons. Animal Behaviour, 35, 387-394.

Gould, J. L. (1974). Honey bee communication. Nature, 252, 300301.

Gould, J. L. (1975a). Communication of distance information by honey bees. Journal of Comparative Physiology, 104, 161-173.

GouLD, J. L. (1975b). Honey bee recruitment: The dance-language controversy. Science, 189, 685-693.

Gould, J. L. (1976). The dance-language controversy. Quarterly Review of Biology, 51, 211-244.

GRIFFIN, A. S. (2004). Social learning about predators: A review and prospectus. Learning \& Behavior, 32, 131-140.

Gruber, T., Muller, M. N., Strimling, P., Wrangham, R., \& ZuBERBÜHLER, K. (2009). Wild chimpanzees rely on cultural knowledge to solve an experimental honey acquisition task. Current Biology, 19, 1806-1810.

GrÜTER, C., \& FARINA, W. M. (2009). The honeybee waggle dance: Can we follow the steps? Trends in Ecology \& Evolution, 24, 242-247.

Hannah, A. C., \& McGrew, W. C. (1987). Chimpanzees using stones to crack open oil palm nuts in Liberia. Primates, 28, 31-46.

Haslam, M., Hernandez-Aguilar, A., Ling, V., Carvalho, S., de la Torre, I., DeStefano, A., ET AL. (2009). Primate archaeology. Nature, 460, 339-344.

HeLbIG, A. J. (1991). Inheritance of migratory direction in a bird species: A cross-breeding experiment with SE- and SW-migrating blackcaps (Sylvia atricapilla). Behavioral Ecology \& Sociobiology, 28, 9-12.

Helfman, G. S., \& Schultz, E. T. (1984). Social transmission of behavioural traditions in a coral reef fish. Animal Behaviour, 32, 379-384.

Heyes, C. M. (1993). Imitation, culture and cognition. Animal Behaviour, 46, 999-1010.

Heyes, C. M. (1994). Social learning in animals: Categories and mechanisms. Biological Reviews, 69, 207-231.

Heyes, C. M., \& Galef, B. G., JR. (1996). Social learning in animals: The roots of culture. San Diego: Academic Press.

Hinde, R. A., \& FisheR, J. (1951). Further observations on the opening of milk bottles by birds. British Birds, 44, 393-396.

HINDE, R. A., \& FISHER, J. (1972). Some comments on the republication of two papers on the opening of milk bottles by birds. In P. H. Klopfer \& J. P. Hailman (Eds.), Function and evolution of behavior: An historical sample from the pen of ethologists (pp. 377-378). Reading, MA: Addison-Wesley. 
Hirata, S., Watanabe, K., \& Kawai, M. (2001). "Sweet-potato washing" revisited. In T. Matsuzawa (Ed.), Primate origins of human cognition and behavior (pp. 487-508). Tokyo: Springer.

Hromada, M., Antczak, M., Valone, T. J., \& Tryjanowski, P. (2008). Settling decisions and heterospecific social information use in shrikes. PLOS ONE, 3, e3930.

Huber, L., Rechberger, S., \& Taborsky, M. (2001). Social learning affects object exploration and manipulation in keas, Nestor notabilis. Animal Behaviour, 62, 945-954.

Humle, T., \& Matsuzawa, T. (2002). Ant-dipping among the chimpanzees of Bossou, Guinea, and some comparisons with other sites. American Journal of Primatology, 58, 133-148.

Hunt, G. R. (1996). Manufacture and use of hook-tools by New Caledonian crows. Nature, 379, 249-251.

Hunt, G. R., \& GraY, R. D. (2004). Direct observations of pandanustool manufacture and use by a New Caledonian crow (Corvus moneduloides). Animal Cognition, 7, 114-120.

INGRAM, J. (1998). The barmaid's brain. Toronto: Viking.

Inoue-Nakamura, N., \& Matsuzawa, T. (1997). Development of stone tool use by wild chimpanzees (Pan troglodytes). Journal of Comparative Psychology, 111, 159-173.

JENKINS, P. F. (1978). Cultural transmission of song patterns and dialect development in a free-living bird population. Animal Behaviour, 25, 50-78.

KAWAI, M. (1965). Newly-acquired pre-cultural behavior of the natural troop of Japanese monkeys on Koshima Islet. Primates, 6, 1-30.

Kendal, R. L., Coolen, I., van Bergen, Y., \& Laland, K. N. (2005). Trade-offs in the adaptive use of social and asocial learning. Advances in the Study of Behavior, 35, 333-379.

Kendal, R. L., Custance, D. M., Kendal, J. R., Vale, G., Stoinski, T. S., Rakotomalala, N. L., \& Rasamimanana, H. (2010). Evidence for social learning in wild lemurs (Lemur catta). Learning \& Behavior, 38, 220-234

Kendal, R. L., Galef, B. G., \& van Schaik, C. P. (2010). Social learning research outside the laboratory: How and why? Learning \& Behavior, 38, 187-194.

Kendal, R. L., Kendal, J. R., Hoppitt, W., \& Laland, K. N. (2009). Identifying social learning in animal populations: A new "optionbias" method. PLoS ONE, 4, e6541.

Kenward, B., Rutz, C., Weir, A. A. S., \& Kacelnik, A. (2006). Development of tool use in New Caledonian crows: Inherited action patterns and social influences. Animal Behaviour, 72, 1329-1343.

Kenward, B., Weir, A. A. S., Rutz, C., \& Kacelnik, A. (2005). Tool manufacture by naive juvenile crows. Nature, $\mathbf{4 3 3}, 121$.

Kothbauer-Hellman, R. (1990). On the origin of a tradition: Milk bottle opening by titmice. Zoologischer Anzeiger, 225, 353-361.

Kummer, H., \& GoOdAll, J. (1985). Conditions of innovative behaviour in primates. Philosophical Transactions of the Royal Society B, 308, 203-214.

LAIDRE, M. E. (2010). How rugged individualists enable one another to find food and shelter: Field experiments with tropical hermit crabs. Proceedings of the Royal Society B, 277, 1361-1369.

LALAND, K. N. (2004). Social learning strategies. Learning \& Behavior, 32, 4-14.

Laland, K. N., \& Galef, B. G. (Eds.) (2009). The question of animal culture. Cambridge, MA: Harvard University Press.

Laland, K. N., \& HoppitT, W. (2003). Do animals have culture? Evolutionary Anthropology, 12, 150-159.

Laland, K. N., \& JANIK, V. M. (2006). The animal cultures debate. Trends in Ecology \& Evolution, 21, 542-547.

Laland, K. N., \& PlotKin, H. C. (1992). Further experimental analysis of the social learning and transmission of foraging information amongst Norway rats. Behavioural Processes, 27, 53-64.

LaLAND, K. N., \& Reader, S. M. (1999). Foraging innovation is inversely related to competitive ability in male but not in female guppies. Behavioral Ecology, 10, 270-274.

Laland, K. N., Richerson, P. J., \& Boyd, R. (1993). Animal social learning: Toward a new theoretical approach. In P. P. G. Bateson, P. H. Klopfer, \& N. S. Thompson (Eds.), Perspectives in ethology: Vol. 10. Behaviour and evolution (pp. 249-277). New York: Plenum.

Laland, K. N., \& Williams, K. (1997). Shoaling generates social learning of foraging information in guppies. Animal Behaviour, 53, 1161-1169.
LANGEN, T. A. (1996). Social learning of a novel foraging skill by white throated magpie jays (Calocitta formosa, Corvidae): A field experiment. Ethology, 102, 157-166.

Leadbeater, E., \& ChittKa, L. (2007). Social learning in insectsFrom miniature brains to consensus building. Current Biology, 17, R703-R713.

Leadbeater, E., \& ChittKa, L. (2009). Bumblebees learn the value of social cues through experience. Biology Letters, 5, 310-312.

LEE, P. (1991). Adaptations to environmental change: An evolutionary perspective. In H. O. Box (Ed.), Primate responses to environmental change (pp. 39-56). London: Chapman \& Hall.

Lefebvre, L. (1986). Cultural diffusion of a novel food-finding behaviour in urban pigeons: An experimental field test. Ethology, $\mathbf{7 1}$ 295-304.

LEFEBVRE, L. (1995). The opening of milk-bottles by birds: Evidence for accelerating learning rates, but against the wave-of-advance model of cultural transmission. Behavioural Processes, 34, 43-53.

Lefebvre, L., \& Giraldeau, L.-A. (1994). Cultural transmission in pigeons is affected by the number of tutors and bystanders present. Animal Behaviour, 47, 331-337.

Lefebvre, L., \& GiraldeAU, L.-A. (1996). Is social learning an adaptive specialization? In C. M. Heyes \& B. G. Galef, Jr. (Eds.), Social learning in animals: The roots of culture (pp. 107-128). San Diego: Academic Press.

Lefebvre, L., \& Palameta, B. (1988). Mechanisms, ecology and population diffusion of socially learned, food-finding behaviour in feral pigeons. In T. Zentall \& B. G. Galef, Jr. (Eds.), Social learning: Psychological and biological perspectives (pp. 141-163). Hillsdale, NJ: Erlbaum.

Lindeyer, C. M., \& Reader, S. M. (2010). Social learning of escape routes in zebrafish and the stability of behavioural traditions. Animal Behaviour, 79, 827-834.

LONSDORF, E. V., \& BonNIE, K. E. (2010). Opportunities and constraints when studying social learning: Developmental approaches and social factors. Learning \& Behavior, 38, 195-205.

Lonsdorf, E. V., Eberly, L. E., \& Pusey, A. E. (2004). Sex differences in learning in chimpanzees. Nature, 428, 715-716.

LYNCH, A., \& BAKER, A. J. (1994). A population memetics approach to cultural evolution in Chaffinch song: Differentiation among populations. Evolution, 48, 351-359.

Matsuzawa, T. (1994). Field experiments on use of stone tools by chimpanzees in the wild. In R. Wrangham, W. McGrew, F. de Waal, \& P. Heltne (Eds.), Chimpanzee cultures (pp. 351-370). Cambridge, MA: Harvard University Press.

Matsuzawa, T., Biro, D., Humle, T., Inoue-Nakamura, N., ToNOOKA, R., \& YAMAKoshi, G. (2001). Emergence of culture in wild chimpanzees: Education by master-apprenticeship. In T. Matsuzawa (Ed.), Primate origins of human cognition and behavior (pp. 557574). Tokyo: Springer.

Matsuzawa, T., \& Yamakoshi, G. (1996). Comparison of chimpanzee material culture between Bossou and Nimba, West Africa. In A. E. Russon, K. Bard, \& S. Parker (Eds.), Reaching into thought: The minds of the great apes (pp. 351-370). Cambridge: Cambridge University Press.

McDougall, P. T., Réale, D., Sol, D., \& Reader, S. M. (2006). Wildlife conservation and animal temperament: Causes and consequences of evolutionary change for captive, reintroduced, and wild populations. Animal Conservation, 9, 39-48.

Michelsen, A., Andersen, B. B., Storm, J., Kirchner, W. H., \& LinDANER, M. (1992). How honeybees perceive communication dances, studied by means of a mechanical model. Behavioral Ecology \& Sociobiology, 30, 143-150

Midford, P. E., Hailman, J. P., \& Woolfenden, G. E. (2000). Social learning of a novel foraging patch in families of free-living Florida scrub-jays. Animal Behaviour, 59, 1199-1207.

Mineka, S., \& CoOK, M. (1988). Social learning and the acquisition of snake fear in monkeys. In T. Zentall \& B. G. Galef, Jr. (Eds.), Social learning: Psychological and biological perspectives (pp. 51-73). Hillsdale, NJ: Erlbaum.

Morand-Ferron, J., Doligez, B., Dall, S. R. X., \& Reader, S. M (in press). Social information use. In M. D. Breed \& J. Moore (Eds.), Encyclopedia of animal behavior. San Diego: Academic Press.

Morand-Ferron, J., Lefebvre, L., Reader, S. M., Sol, D., \& 
Elvin, S. (2004). Dunking behaviour in Carib grackles. Animal Behaviour, 68, 1267-1274.

Mukhin, A., Kosarev, V., \& Ktitorov, P. (2005). Nocturnal life of young songbirds well before migration. Proceedings of the Royal Society $B, 272,1535-1539$.

Müller, C. A., \& CANT, M. A. (in press). Imitation and traditions in wild banded mongooses. Current Biology.

Nakamura, M., \& Uehara, S. (2004). Proximate factors of different types of grooming hand-clasp in Mahale chimpanzees: Implications for chimpanzee social customs. Current Anthropology, 45, 108-114.

Nicol, C. J., \& Pope, S. J. (1994). Social learning in small flocks of laying hens. Animal Behaviour, 47, 1289-1296.

Noble, J., Todd, P. M., \& Tucit, E. (2001). Explaining social learning of food preferences without aversions: An evolutionary simulation model of Norway rats. Proceedings of the Royal Society B, 268, 141149

Nocera, J. J., Forbes, G. J., \& Giraldeau, L.-A. (2006). Inadvertent social information in breeding site selection of natal dispersing birds. Proceedings of the Royal Society B, 273, 349-355.

Nordby, J. C., CAmpbell, S. E., Burt, J. M., \& Beecher, M. D. (2000). Social influences during song development in the song sparrow: A laboratory experiment simulating field conditions. Animal Behaviour, 59, 1187-1197.

Odling-Smee, F. J., Laland, K. N., \& Feldman, M. W. (2003). Niche construction: The neglected process in evolution. Princeton, NJ: Princeton University Press.

Orenstein, R. I. (1972). Tool use by the New Caledonian crow, Corvus moneduloides. Auk, 89, 674-676.

Plotkin, R. T., Byles, R. A., Rostal, D. C., \& Owens, D. W. (1995). Independent versus socially facilitated oceanic migrations of the olive ridley, Lepidochelys olivacea. Marine Biology, 122, 137-143.

READER, S. M. (2004). Distinguishing social and asocial learning using diffusion dynamics. Learning \& Behavior, 32, 90-104.

Reader, S. M., Kendal, J. R., \& Laland, K. N. (2003). Social learning of foraging sites and escape routes in wild Trinidadian guppies. Animal Behaviour, 66, 729-739.

Reader, S. M., \& Laland, K. N. (2001). Primate innovation: Sex, age and social rank differences. International Journal of Primatology, 22, 787-805.

Reader, S. M., \& Laland, K. N. (2002). Social intelligence, innovation and enhanced brain size in primates. Proceedings of the National Academy of Sciences, 99, 4436-4441.

Reader, S. M., \& Laland, K. N. (Eds.) (2003a). Animal innovation. Oxford: Oxford University Press.

Reader, S. M., \& LALAND, K. N. (2003b). Animal innovation: An introduction. In S. M. Reader \& K. N. Laland (Eds.), Animal innovation (pp. 3-35). Oxford: Oxford University Press.

ReAder, S. M., \& Lefebvre, L. (2001). Social learning and sociality. Behavioral \& Brain Sciences, 24, 353-355.

Reader, S. M., Nover, D., \& Lefebvre, L. (2002). Locale-specific sugar packet opening by Lesser Antillean bullfinches in Barbados. Journal of Field Ornithology, 73, 82-85.

SEPPÄNEn, J.-T., \& Forsman, J. T. (2007). Interspecific social learning: Novel preference can be acquired from a competing species. Current Biology, 17, 1248-1252.

Sherman, G., \& Visscher, P. K. (2002). Honeybee colonies achieve fitness through dancing. Nature, 419, 920-922.

Sherry, D. F., \& GALEF, B. G., JR. (1984). Cultural transmission without imitation: Milk bottle opening by birds. Animal Behaviour, 32, 937-938.

Sherry, D. F., \& GAleF, B. G., JR. (1990). Social learning without imitation: More about milk bottle opening by birds. Animal Behaviour, 40, 987-989.

SHIER, D. M., \& Owings, D. H. (2007). Effects of social learning on predator training and postrelease survival in juvenile black-tailed prairie dogs, Cynomys ludovicianus. Animal Behaviour, 73, 567-577.

Shimada, M. K., Hayakawa, S., Humle, T., Fujita, S., Hirata, S., SugiYama, Y., \& Saitou, N. (2004). Mitochondrial DNA genealogy of chimpanzees in the Nimba Mountains and Bossou, West Africa. American Journal of Primatology, 64, 261-275.

Slabbekoorn, H., \& Ripmeester, E. A. P. (2008). Birdsong and an- thropogenic noise: Implications and applications for conservation. Molecular Ecology, 17, 72-83.

Slagsvold, T., \& WieBe, K. L. (2007). Learning the ecological niche. Proceedings of the Royal Society B, 274, 19-23.

Slater, P. J. B. (1986). The cultural transmission of bird song. Trends in Ecology \& Evolution, 1, 94-97.

Sol, D., Bacher, S., Reader, S. M., \& Lefebvre, L. (2008). Brain size predicts the success of mammal species introduced into novel environments. American Naturalist, 172, S63-S71.

Sol, D., Lefebvre, L., \& Timmermans, S. (2002). Behavioural flexibility and invasion success in birds. Animal Behaviour, 63, 495-502.

Stoddard, P. K., Beecher, M. D., Homing, C. L., \& Campbell, S. E. (1991). Recognition of individual neighbors by song in the song sparrow, a species with song repertoires. Behavioral Ecology \& Sociobiology, 29, 211-215.

Suboski, M. D., \& Templeton, J. J. (1989). Life skills training for hatchery fish: Social learning and survival. Fisheries Research, 7, 343-352.

Tebbich, S., Taborsky, M., Fessl, B., \& Blomevist, D. (2001). Do woodpecker finches acquire tool-use by social learning? Proceedings of the Royal Society B, 268, 2189-2196.

Templeton, C. N., AKÇay, Ç., Campbell, S. E., \& Beecher, M. D. (2010). Juvenile sparrows preferentially eavesdrop on adult song interactions. Proceedings of the Royal Society B, 277, 447-453.

Templeton, J. J., Kamil, A. C., \& Balda, R. P. (1999). Sociality and social learning in two species of corvids: The pinyon jay (Gymnorhinus cyanocephalus) and the Clark's nutcracker (Nucifraga columbiana). Journal of Comparative Psychology, 113, 450-455.

Terkel, J. (1996). Cultural transmission of feeding behaviour in the black rat (Rattus rattus). In C. M. Heyes \& B. G. Galef, Jr. (Eds.), Social learning in animals: The roots of culture (pp. 17-48). London: Academic Press

Thornton, A. (2008). Social learning about novel foods by young meerkats. Animal Behaviour, 76, 227-229.

Thornton, A., \& Malapert, A. (2009). The rise and fall of an arbitrary tradition: An experiment with wild meerkats. Proceedings of the Royal Society B, 276, 1269-1276.

Thornton, A., \& McAuliffe, K. (2006). Teaching in wild meerkats. Science, 313, 227-229.

Thornton, A., \& Raihani, N. J. (2010). Identifying teaching in wild animals. Learning \& Behavior, 38, 297-309.

TINBERGEN, N. (1963). On aims and methods of ethology. Zietschrift für Tierpsychologie, 20, 410-433.

Toelch, U., van Delft, M. J., Bruce, M. J., Donders, R., Meeus, M. T. H., \& READER, S. M. (2009). Decreased environmental variability induces a bias for social information use in humans. Evolution \& Human Behavior, 30, 32-40.

VAN DER Post, D. J., \& Hogeweg, P. (2006). Resource distributions and diet development by trial-and-error learning. Behavioral Ecology \& Sociobiology, 61, 65-80.

van de WaAl, E., Renevey, N., Favre, C. M., \& Bshary, R. (2010). Selective attention to philopatric models causes directed social learning in wild vervet monkeys. Proceedings of the Royal Society B, 277, 2105-2111.

VAN SCHAIK, C. P. (2002). Fragility of traditions: The disturbance hypothesis for the loss of local traditions in orangutans. International Journal of Primatology, 23, 527-538

van Schaik, C. P., Ancrenaz, M., Borgen, G., Galdikas, B., Knott, C. D., Singleton, I., ET AL. (2003). Orangutan cultures and the evolution of material culture. Science, 299, 102-105.

VisalberGhi, E., \& Fragaszy, D. M. (1990). Food-washing behaviour in tufted capuchin monkeys, Cebus apella, and crabeating macaques, Macaca fascicularis. Animal Behaviour, 40, 829-836.

WARNER, R. R. (1988). Traditionality of mating-site preferences in a coral reef fish. Nature, 335, 719-721.

WARNER, R. R. (1990a). Male versus female influences on mating-site determination in a coral-reef fish. Animal Behaviour, 39, 540-548.

WARNER, R. R. (1990b). Resource assessment versus tradition in matingsite determination. American Naturalist, 135, 205-217.

Weigl, P. D., \& Hanson, E. V. (1980). Observational learning and the feeding behavior of the red squirrel Tamiasciurus hudsonicus: The ontogeny of optimization. Ecology, 61, 214-218. 
Whitehead, H. (2010). Conserving and managing animals that learn socially and share cultures. Learning \& Behavior, 38, 329-336.

Whiten, A., Goodall, J., McGrew, W. C., Nishida, T., ReYnolds, V., SugiYama, Y., ET AL. (1999). Cultures in chimpanzees. Nature, 399, 682-685.

Whiten, A., Goodall, J., McGrew, W. C., Nishida, T., Reynolds, V., SugiYama, Y., ET AL. (2001). Charting cultural variation in chimpanzees. Behaviour, 138, 1481-1516.

Whiten, A., Horner, V., \& De WaAl, F. B. M. (2005). Conformity to cultural norms of tool use in chimpanzees. Nature, 437, 737-740.

Whiten, A., \& Mesoudi, A. (2008). Establishing an experimental science of culture: Animal social diffusion experiments. Philosophical Transactions of the Royal Society B, 363, 3477-3488.

Whiten, A., Spiteri, A., Horner, V., Bonnie, K. E., Lambeth, S. P., Schapiro, S. J., \& DE WAAL, F. B. M. (2007). Transmission of multiple traditions within and between chimpanzee groups. Current Biology, 17, 1038-1043.

WitTe, K., \& RYAN, M. J. (2002). Mate choice copying in the sailfin molly, Poecilia latipinna, in the wild. Animal Behaviour, 63, 943-949.

Wyles, J. S., Kunkel, J. G., \& Wilson, A. C. (1983). Birds, behaviour, and anatomical evolution. Proceedings of the National Academy of Sciences, 80, 4394-4397.

ZANN, R. (1990). Song and call learning in wild zebra finches in southeast Australia. Animal Behaviour, 40, 811-828.

\section{NOTES}

1. Humle and Matsuzawa (2002) involved no explicit manipulation of the environment or of individuals.

2. Witte and Ryan (2002) had no test of learning.

3. Matsuzawa et al. (2001) introduced unfamiliar nuts to a nutcracking population of wild chimpanzees, but the extent to which a suspected model was responsible for the acceptance of the nuts by the rest of the community is unclear in the absence of a control population with no demonstrator

4. Multiple studies on the same behavior pattern in the same species are counted once.

(Manuscript received March 22, 2010; accepted for publication May 6, 2010.) 